\title{
Occupational Hazards and Social Disability Insurance
}

Working Paper 2017-11

Amand Michaud AND DAVID WiCZER

October 18, 2017

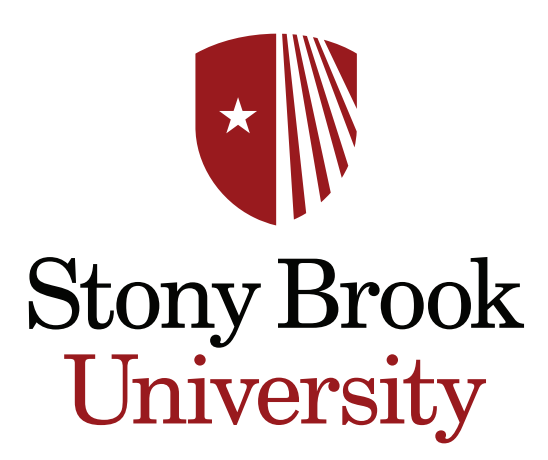




\title{
Occupational Hazards and Social Disability Insurance
}

\author{
Amanda Michaud ${ }^{\mathrm{a}, *}$, David Wiczer ${ }^{\mathrm{b}}$ \\ ${ }^{a}$ Indiana University \\ ${ }^{b}$ Stony Brook Univeristy and FRB St Louis
}

\begin{abstract}
Using retrospective data, we introduce evidence that occupational exposure significantly affects disability risk. Incorporating this into a general equilibrium model, social disability insurance (SDI) affects welfare through (i) the classic, risk-sharing channel and (ii) a new channel of occupational reallocation. Both channels can increase welfare, but at the optimal SDI they are at odds. Welfare gains from additional risk-sharing are reduced by overly incentivizing workers to choose risky occupations. In a calibration, optimal SDI increases welfare by $2.3 \%$ relative to actuarially fair insurance, mostly due to risk sharing.
\end{abstract}

JEL CODES: E62, I13

Keywords: Disability Insurance, Occupational Choice, Optimal Policy

*Corresponding author; E-mail: amanda.michaud@gmail.com; Phone: 1-(812)-8561238; Mailing address: Department of Economics, Indiana University; 100 S. Woodlawn Ave, Bloomington, IN 47405-7104. We thank Victor Rios-Rull, Juan-Carlos Hatchondo, Jonathan Heathcote, John Kennan, Sam Schulhofer-Wohl, and Ali Shorideh; workshop participants at University of Illinois and University of California-Santa Barbara, and seminars at the Bureau of Labor Statistics, Notre Dame, Iowa, Washington University in St. Louis, and Windsor. James Eubanks provided excellent research assistance. The views expressed herein are those of the authors and do not necessarily reflect the views of the Federal Reserve System, the Boards of Governors, or the regional Federal Reserve Banks. 


\section{Introduction}

This paper considers how workers' occupational choice affects the optimality of social - or public - disability insurance (SDI). The two are related because, in choosing their occupation, workers are choosing their physical health risk and, hence, their likelihood to need disability insurance. Disability insurance then, alters the composition of occupations by incentivizing workers to chose occupations with higher risk. This reallocation towards risky occupations can, however, be desirable when occupations are imperfect substitutes in production and insurance markets are incomplete.

Our central results are based on a theoretical model of occupation choice, occupation-specific disability risk, and incomplete markets for private insurance. We show that fewer workers choose occupations with high disability risk than would achieve productive efficiency. This occurs because workers in high-risk jobs demand a compensating differential, a wage sufficient to selfinsure against disability. But with a downward sloping demand for additional workers in each occupation, these high wages obtain only if there are inefficiently few workers in high-risk occupations. In other words, self-insurance is too expensive and so equilibrium prices correspond to an inefficient distribution of workers.

The introduction of SDI improves welfare through two channels. The first is through risk sharing: social insurance improves welfare by helping workers in risky occupations to smooth consumption. The second, novel channel is occupational reallocation: SDI encourages more workers to choose risky occupations. On the margin, the allocation of workers across occupations becomes more efficient and output increases. The gains are larger than the cost to fund the scheme because social insurance is more efficient than selfinsurance. With self-insurance, some workers who accumulate savings never become disabled. The increase in output provides welfare gains even for workers with zero disability risk and many whose disability risk never realizes.

The two channels, risk-sharing and the reallocation, both work to increase 
welfare upon introduction of social insurance, but at the welfare-maximizing level they are at odds. Although reallocation towards risky occupations initially improves productive efficiency, additional insurance can increase the risky occupational allocation beyond the output maximizing level. At the point at which an SDI expansion reduces output, welfare gains from risksharing remain. This result is general and not a quantitative statement; welfare is maximized by an SDI program that induces workers to choose risky occupations beyond the output maximizing level.

Our results are qualitatively robust to ex ante heterogeneity in preferences that induce sorting and to private information over disability status, though this imposes bounds on the benefit. Further, private information over disability status is a reason for why private insurance cannot achieve the same welfare gains as social insurance. If neither public nor private contracts can condition on workers' risk-level, i.e. contracts are not occupation-specific, high-risk workers over-subscribe to private insurance contracts. This adverse selection leads to a market failure typical of insurance contracts with private information. ${ }^{1}$ Social insurance can dictate equal insurance coverage so it is not subject to adverse selection and always generates welfare gains.

To motivate our theoretical work, we show heterogeneity in disability incidence across occupations. Using data from the University of Michigan's Health and Retirement Survey (HRS), we find a natural grouping between low- and high-risk occupations, the latter have about twice the disability rate as the low-risk group. While this is suggestive, occupational choice is endogenous and potentially influenced by unobservable factors. To begin addressing this, we propose an instrument scheme using $\mathrm{O}^{*}$ NET measures of physical and non-physical occupational requirements. Physical requirements

\footnotetext{
${ }^{1}$ Market failure here is Rothschild Stiglitz-like, in that if an insurance contract were offered, it would attract high-risk workers and earn a negative profit. Increasing the price only makes adverse selection worse.
} 
have health repercussions, but their estimated effect may be influenced by sorting along these physical requirements. Therefore, we instrument physical requirements by the non-physical requirements in the $\mathrm{O}^{*} \mathrm{NET}$. The intent is to use how occupations bundle requirements: While workers' unobserved physical traits may guide sorting along the physical requirements, they hopefully do not guide sorting on non-physical requirements, but, these requirements can predict the physical requirements. This instrumenting scheme is not a definitive solution, but it does uncover significant bias in the estimate assuming exogeneity.

We use these facts, along with the US SSDI system, to calibrate our model of occupation risk. We find the optimal SDI program costs $4.8 \%$ of GDP and provides welfare gains equivalent to a $2.3 \%$ increase in consumption in a world with actuarially fair insurance alone. Relative to this optimal program, the current US system captures $33 \%$ of the potential gains. We conclude from these findings that there is a quantitatively important role for SDI beyond the insurance that is provided by private markets.

The economic mechanism of this paper is most related to that of Acemoglu and Shimer (1999). They show unemployment insurance can raise output by inducing workers to search for higher productivity jobs which are rarer, and therefore, more risky to pursue. In this paper, SDI also increases output by inducing workers to take on more risk in their job search; specifically choosing occupations with greater disability risk. In both, social insurance is not simply a transfer to those experiencing bad luck. Instead, improvements in productive efficiency increase the welfare of all individuals, even those who face little or no risk.

Schulhofer-Wohl (2011) also considers workers who choose jobs with different levels of risk and focuses on underlying heterogeneity in preferences. He shows that this generalization can reduce the welfare costs associated with incomplete insurance. In an extension, we incorporate preference heterogeneity and show our SDI scheme still generates Pareto-improving welfare 
gains, although those gains may now be unequal. Quite consistent with the results of Schulhofer-Wohl (2011) Workers in the most risky occupations may gain the least from insurance because the most impatient agents select into those occupations.

Several papers discuss disability's interaction with other economic factors. Notably, Golosov and Tsyvinski (2006) also consider optimal DI, but from a mechanism design approach to prevent misreporting. This consideration is important, and so we show workers reveal their status in our baseline SDI scheme because our policy tool is already constrained from providing full-insurance.

We motivate our normative work by estimating the heterogeneity in disability risk across occupations. A few papers present similar empirical results (e.g. Fletcher et al., 2009; Morefield et al., 2011). These papers both connect physically demanding occupations to health problems later in life. Ravesteijn et al. (2013) use German data and, again, link occupational physical demands to health deteriorations. They use a dynamic fixed effects model to control the individual effects affecting both occupational choice and health outcomes. We introduce a set of instrumental variable techniques to specifically address this problem of endogeneity between occupational choice and potential heterogeneity in risk sensitivity.

\section{Data on occupations and disability}

In this section, we present data regarding the connection between an individual's occupation and disability risk. First, we construct a measure of lifetime exposure to an occupation using the University of Michigan's Health and Retirement Study (HRS). The survey respondents provide detailed information on their health conditions, from which we infer whether they are "disabled." We find that occupations have quite disparate disability rates, much of which can be attributed to their occupations' effects themselves. 


\subsection{Constructing the dataset}

We begin with RAND's distribution of the HRS with some restrictions described in A.1. We use one of two measures of disability for health problems during working life, before 65 . Our baseline uses the more restrictive measure, which looks at whether they report limitations to activities of daily living (ADLs). The broader of the two uses the survey's direct question, whether a health problem limits one's ability to do paid work. Results are generally very similar between the two, as shown in the online Appendix.

We will associate an individual with his or her longest-held occupation, reported retrospectively. If we used the current occupation, we would mistakenly consider an occupation dangerous if workers in poor health switched into that occupation later in life. For each occupation, we merge in Knowledge, Skills and Abilities descriptors from O*NET version 4.0, "analyst," database. We then use the first principal component from the 19 physical descriptors and three components from the 101 non-physical descriptors.

\subsection{The distribution of disability across occupations}

Figure 1 shows the distribution across occupations of the incidence of a disability, as measured by having a difficulty with an ADL. In this kernel density estimate, each data point is an occupation, and within that occupation, we construct the average rate of ADL difficulties. We then weight the occupations by their population using the appropriate weights provided by the HRS. Along the horizontal axis, we plot dots representing the position of occupations.

[Figure 1 about here.]

Observationally, there are two clusters of occupations, a larger low-incidence group and a tail with more than twice the average rate of ADL difficulties. To summarize these two groups, we estimate a mixture of two normal distributions to classify occupations as "risky" or "safe." From our primary 
measure, whether a worker has any limitations to ADLs, the mean disability rate in the high risk group is about $16 \%$, twice that of the the low-risk group. About $17 \%$ of the population worked in this high risk group.

The largest high-risk occupations are machine and transport operators and also include construction and extraction occupations. The large, low-risk occupations are professional and management occupations, administrative support and sales.

\subsection{Demonstrative evidence of the occupation effect}

How much of the difference in disability outcomes across occupations can be ascribed to the occupations themselves? Potentially, there are systematic differences between individuals who choose what appear to be "risky" occupations and those who choose safe occupations. We first use an Oaxaca-Blinder decomposition to extract the residual effect of occupation on disability from observable differences between workers in different occupations. However, it is problematic to interpret this decomposition because the grouping is endogenous, i.e. people choose their occupations. We attempt to address the issue of endogenous occupation selection by introducing an instrument: We use $\mathrm{O}^{*}$ NET measures of physical demands to summarize the occupation's effect on health, which we instrument by the non-physical demands that tend to be bundled together within occupations.

The Oaxaca-Blinder decomposition splits the differences in disability outcomes between observable characteristics and residual differences in disability rates across occupation groups. We group occupations into two sets, low- and high-risk, based on the mixture we estimated on Figure 1, assigning each occupation to the distribution with highest density at its level of risk. Then we regress an indicator of whether the individual ever reported disability on the following regressors: a cubic for potential experience, body mass index (BMI), time, dummies for education level, gender, marital status, race, and tobacco use. We set the characteristics of workers who report a disability by their values in the wave in which they become disabled. For workers who 
never report a disability, we use the mean value of their characteristics across waves. The results of the decomposition are summarized in Table 1.

[Table 1 about here.]

Column (1) of Table 1 averages covariates to make one observation per individuals; column (2) use multiple periods per individual. In our baseline case, Column (1), the occupation effect accounts for $42 \%$ of the difference in risk. This decomposition suggests much of the difference in risk is unexplained by population differences without differences in occupations themselves.

While this decomposition is instructive, there may also be unobservable characteristics that differ across workers in risky and safe occupations, and this implies there also may be sorting. For example, if more physically robust workers sort into physically-demanding occupations, this would attenuate occupations' effect. We now use instruments to partly address this.

We map occupations' health risk into the physical demands characterized by the $\mathrm{O}^{*} \mathrm{NET}$, the construction of which we described in subsection 2.1. To relate to our two occupation groups, risky occupations have a mean physical requirement of 1.05 and safe occupations have a mean of -0.65 , where the population is mean 0 and standard deviation one. Using a direct measure of physical requirement instead of the identity of the occupation cuts away other characteristics of occupations that may be related to disability, such as economic decline. However, it does not address sorting on the desired level of health risk itself, the fundamental endogeneity problem we face.

To address this, we create a set of instrumental variables from the nonphysical requirements of occupations. Because occupations bundle many requirements, and there are patterns in these bundles, we can use the nonphysical requirements to predict the physical requirements of an occupation. These requirements will be valid instruments if non-physical requirements are uncorrelated with one's propensity for disability. 
To make clear our strategy, for an individual $i$ in occupation $j$, we model the probability of ever becoming disabled before age 65 as

$$
\operatorname{Pr}\left[\operatorname{Disabled}_{i}\right]=f\left(\gamma H_{j}+x_{i}^{\prime} \beta+\nu_{i}\right)
$$

where $x_{i}$ is the same vector of observable characteristics as our previous specification and $f$ is the link function. $H_{j}$ is the first principal component of the physical descriptors associated with occupation $j$ and $\nu_{i}$ is an unobservable individual trait, physical "robustness." Robustness may affect both the probability of disability and the level of physical requirements one chooses in an occupation. We therefore treat $H_{j}$ as endogenous and instrument it with the first 3 principal components of the non-physical requirements of the occupation. $^{2}$ The idea behind our instrument is that workers may choose their occupation considering $\nu_{i}$, e.g. particularly athletic individuals may chose more physically intense jobs given they know they are less sensitive to these demands, but they do not chose other non-physical occupational characteristics based on $\nu_{i}$. Occupations, however, follow certain patterns in how they bundle requirements, so the non-physical requirements can predict well the physical requirements.

The marginal effects from the estimation of Equation 2.1 are in Table 2. Column (1) displays the baseline IV-Probit estimates. Column (2) adds industry fixed effects and clusters standard errors on industry, which may be important to control for economic factors affecting labor-force attachment and disability reporting. Column (3) uses health-limitations rather than ADL difficulties as the measure of disability. Columns (4)-(6) repeat these specifications but without instrumenting. The first stage of the IV,

\footnotetext{
${ }^{2}$ The number of components we use as instruments is not completely innocuous. Doubling the number of components, they predict the physical requirements near perfectly and invalidate the instruments. Our results are qualitatively the same if we increase the number of principal components to 4 or reduce to 2 .
} 
in Appendix Section A.2 which shows that non-physical requirements are strongly negatively related to physical requirements. To depart from distributional assumptions of the Probit, we also include estimates of the linear probability model and IV linear probability model in A.3. The coefficients are quantitatively very similar to the marginal effects of our Probit.

[Table 2 about here.]

The principal result from these estimates is in the first row and is quite consistent across estimation methods. In our baseline specification, a standard deviation change in physical requirements increases disability risk by 3.4 percentage points. Applying this coefficient to our two groups of occupations: the higher physical requirement of risky occupations implies about a 5.7 percentage point higher rate of ADL difficulty than other occupations. Notice that the instruments increase the margin by a small, but statistically significantly different amount, which suggests that people sort into more physically demanding occupations if they have a lower disability risk.

The results of our instrumental scheme are suggestive of the underlying relationship on which our theory is based; however, one could reasonably question the validity of these instruments. Essentially, if traits are correlated then sorting on physical robustness also implies sorting on non-physical acuity, the instrument will be invalid. Further, the actual task and skill composition of occupations are endogenous to a degree: the bundle is chosen in part as a result of the sorting of workers. Quantitatively, the first-stage is worryingly predictive - its F-statistic's p-value is 0.000 - suggesting our instruments are too tightly related to the endogenous variable. So here we have not definitively established a causal link but offered evidence of a relationship.

\section{A Model Economy with Occupation-Specific Disability Risk}

To prove our theoretical results, we use a two-period overlapping generations model where disability status is a publicly-observed, discrete event. 
Workers who receive a disability shock cannot work.

Time is discrete. There is a single consumption good produced with labor by workers in a continuum of different occupations $j \in[0, J]$. The production technology exhibits constant elasticity of substitution across occupations with elasticity of substitution $\rho=\frac{1}{1-\gamma}<\infty$. The index of an occupation also defines an occupation-specific probability of disability in the second period: $\theta(j)=j$.

There is a continuum of competitive firms. The representative firm hires labor $n(j)$ in occupation specific spot markets to solve the following maximization problem: ${ }^{3}$

$$
\begin{gathered}
\max _{\{n(j)\}_{j=0}^{J}} y-\int_{j=0}^{J} w(j) n(j) d j \text { s.t. } \\
y=\left(\int_{j=0}^{J} n(j)^{\gamma}\right)^{1 / \gamma}
\end{gathered}
$$

Each period a unit measure of workers is born. Workers are identical at birth and live for two periods. They have strictly risk-averse, time-separable preferences over consumption in both periods $U\left(c_{1}, c_{2}\right)=u\left(c_{1}\right)+u\left(c_{2}\right)$. The utility function $u(\cdot)$ is assumed to be strictly increasing, strictly concave, homothetic, and continuously differentiable.

In the first period, workers choose a single occupation, which persists their entire lifetime. They then work, collecting wage earnings net of taxes $(1-\tau) w(j)$. From these earnings, they decide how much to consume, $c_{1}$, and how much to save in $a$, a storage technology with rate of return 1 .

In the second period, disability shocks occur with occupation-specific probability $\theta(j)$. Individuals who receive a disability shock cannot work and report themselves as disabled. Their income is disability benefits with

\footnotetext{
${ }^{3}$ Because firms hire in spot markets, their problem is static. This means they cannot design complicated, multi-period wage contracts. It also implies firms do not internalize that hiring workers in risky occupations decreases labor available in the next period.
} 
replacement rate $b$; ie: $b w(j)$. Individuals who do not receive a disability shock will work. Their income is wage earnings net of taxes $(1-\tau) w(j)$. Agents consume whatever income plus savings they have, then die. Subscript 1 denotes period 1 consumption, $d$ is period 2 consumption when a worker is disabled and $n$ is for period 2 when a worker is not disabled. This problem can be represented as $\max _{j \in[0, J]} E_{j}\left[U\left(c_{1}^{*}(j), c_{n}^{*}(j), c_{d}^{*}(j)\right)\right]$, where we use $E_{j}$ to show the expectation over risks associated with occupation $j, \theta(j)$.

$$
\begin{aligned}
E_{j}\left[U\left(c_{1}^{*}(j), c_{n}^{*}(j), c_{d}^{*}(j)\right)\right]= & \max _{c_{1}, c_{n}, c_{d}, a} u\left(c_{1}\right)+(1-\theta(j)) u\left(c_{n}\right)+\theta(j) u\left(c_{d}\right) \text { s.t. } \\
& c_{1} \leq w(j)(1-\tau)-a \\
& c_{n} \leq w(j)(1-\tau)+a \\
& c_{d} \leq a+b w(j) \\
& a \geq 0
\end{aligned}
$$

The solution to this problem gives occupation-specific decision rules: $c_{1}^{*}(j), c_{d}^{*}(j), c_{n}^{*}(j), a^{*}(j)$. Define $\ell^{*}(j)$ as the measure of workers choosing occupation $j$.

Definition 3.1 (Competitive equilibrium). An equilibrium consists of allocations

$\left\{c_{1}^{*}(j), c_{d}^{*}(j), c_{n}^{*}(j), a^{*}(j), \ell^{*}(j), n^{*}(j)\right\}$, government policies $\{\tau, b\}$, and prices $w^{*}(j)$ for every $j \in[0, J]$ such that (i) given prices and government policies, allocations solve the workers' and firms' problems; (ii) feasibility is satisfied in the labor market: $n^{*}(j) \leq(2-\theta(j)) \ell^{*}(j)$; markets clear in (iii) goods; and (iv) government budgets balance period-wise.

Optimality in the labor market requires satisfying two conditions:

- Firms: Wage equals marginal product: $w(j)=\left(\frac{y}{n(j)}\right)^{1-\gamma}$

- Workers: Indifferent between entering any occupation:

$$
\left.E_{j}\left[U\left(c_{1}^{*}(j), c_{n}^{*}(j), c_{d}^{*}(j)\right)\right]=E_{k}\left[U c_{1}^{*}(k), c_{n}^{*}(k), c_{d}^{*}(k)\right)\right] \forall j, k \in[0, J] .
$$


Goods market clearing requires that

$$
y \geq \int_{j=0}^{J} c_{1}(j)+(1-\theta(j)) c_{n}(j)+\theta(j) c_{d}(j) d j
$$

Government budget balance requires

$$
b^{*} \leq \frac{\tau y}{\int_{j} \theta(j) \ell(j)^{*} w(j)^{*} d j}
$$

\subsection{Sources of Inefficiency in the Competitive Equilibrium}

Our first proposition shows that the competitive allocation without SDI $(\tau=0)$ is Pareto inefficient. A first-best social planner can choose an alternative feasible allocation that improves welfare for all agents.

Proposition 3.2. Let $\left\{c_{1}^{c m}(j), c_{d}^{c m}(j), c_{n}^{c m}(j), \ell^{c m}(j)\right\}$ be the first-best planner's allocation. It solves

$$
\begin{aligned}
\max _{\left\{c_{1}(j), c_{d}(j), c_{n}(j), \ell(j)\right\}} & \int_{j} \ell(j)\left(u\left(c_{1}(j)\right)+\theta(j) u\left(c_{d}(j)\right)+(1-\theta(j)) u\left(c_{n}(j)\right)\right) d j \text { s.t. } \\
\left(\int_{j}((2-\theta(j)) \ell(j))^{\gamma} d j\right)^{\frac{1}{\gamma}} & \geq \int_{j} \ell(j)\left(c_{1}(j)+\theta(j) c_{d}(j)+(1-\theta(j)) c_{n}(j)\right) d j \\
1 & \geq \int_{j} \ell(j) d j
\end{aligned}
$$

Then, $\left\{c_{1}^{c m}(j), c_{d}^{c m}(j), c_{n}^{c m}(j), \ell^{c m}(j)\right\}$ strictly Pareto dominates $\left\{c_{1}^{*}(j), c_{d}^{*}(j), c_{n}^{*}(j), \ell^{*}(j)\right\}$.

Proof. See Appendix B.4

The first-best planner's allocation provides welfare gains through two channels: (i) reallocation of consumption across workers (risk-sharing) and (ii) reallocation of workers across occupations. The existence of welfare gains through the first channel is unsurprising: workers have insufficient assets needed to span the risks they face. We formally show its existence within our model in the next proposition. To do so, we fix the labor allocation and output from the competitive equilibrium and provide an alternative feasible consumption allocation that Pareto dominates the competitive allocation. 
Proposition 3.3 (The Competitive Allocation of Consumption Without Insurance is Pareto Inefficient). Let $\left\{c_{1}^{*}(j), c_{n}^{*}(j), c_{d}^{*}(j), a^{*}(j), n^{*}(j), \ell^{*}(j)\right\}$ satisfy Definition 3.1 for the case $b=\tau=0$. There exists an alternative feasible allocation $\left\{\hat{c}_{1}(j), \hat{c}_{d}(j), \hat{c}_{n}(j), \hat{\ell}(j)\right\}$ that:

(i) increases expected utility in each occupations

$$
\begin{aligned}
& E_{j}\left[U\left(\hat{c}_{1}(j), \hat{c}_{n}(j), \hat{c}_{d}(j)\right)\right] \geq E_{j}\left[U\left(c_{1}^{*}(j), c_{n}^{*}(j), c_{d}^{*}(j)\right)\right] \forall j \in[0, J] \\
\exists k \text { s.t. } & E_{k}\left[U\left(\hat{c}_{1}(k), \hat{c}_{n}(k), \hat{c}_{d}(k)\right)\right]>E_{k}\left[U\left(c_{1}^{*}(k), c_{n}^{*}(k), c_{d}^{*}(k)\right)\right]
\end{aligned}
$$

(ii) is feasible

$$
\int_{j} \hat{\ell}(j)\left(\hat{c}_{1}(j)+\theta(j) \hat{c}_{d}(j)+(1-\theta(j)) \hat{c}_{n}(j)\right) d j \leq\left(\int_{j}(\hat{\ell}(j)(2-\theta(j)))^{\gamma} d j\right)^{\frac{1}{\gamma}}
$$

Proof. See Appendix B.2

The next proposition formalizes the existence of the second welfare improving channel in our model: reallocation of labor to improve production efficiency and increase output. With constant-elasticity of substitution (CES) production (indeed, even with linear, $\gamma=1$ ), efficient production requires the marginal product to be constant across occupations. This implies a constant life-time income across occupations. However, with incomplete markets, risk adverse workers require a wage premium to work in more risky occupations relative to less risky. For the competitive allocation to provide this risk premium, fewer workers must choose risky occupations than the efficient, output maximizing allocation. In other words, the competitive allocation of labor across occupations is first-order stochastically dominated by the efficient allocation.

Proposition 3.4. [The competitive allocation without insurance puts too few workers in risky occupations] Let $\ell^{*}(j)$ satisfy Definition 3.1 for the case $b=\tau=0$. Let $\ell^{c m}(j)$ be the feasible, output-maximizing allocation. Then,

$$
\int_{j=0}^{t} \ell^{*}(j) d j \leq \int_{j=0}^{t} \ell^{c m}(j) d j \quad \forall t \in(0, J]
$$


This is to say, the efficient distribution of labor across occupations first-order stochastically dominates the distribution in the competitive allocation.

Proof. See Appendix B.4

The degree of productive inefficiency in the competitive equilibrium depends on both the extent of risk aversion of workers and the elasticity of substitution across occupations in production. As risk aversion increases, the competitive allocation becomes less efficient by concentrating even more workers in less-risky occupations. Comparably, if workers are risk neutral, the competitive allocation is efficient. Figure 2 illustrates this for constant relative risk aversion (CRRA) preferences, $u(c)=\frac{c^{1-\sigma}}{1-\sigma}$, and $\gamma=0.5$.

[Figure 2 about here.]

As the elasticity of substitution between occupations increases, both the competitive and the efficient allocations place fewer workers in the risky occupations. While the efficient allocation always places more workers in the risky occupations, the distance between the two distributions is nonmonotone. When occupations are perfect substitutes, the distributions are equivalent with all of the workers in the safest occupation. When production is Leontief, both allocations evenly distribute workers across occupations. Figure 3 illustrates this, fixing risk aversion at $\sigma=2$.

[Figure 3 about here.]

\subsection{How Social Disability Insurance Improves Welfare}

We have shown that the competitive equilibrium without an SDI scheme is inefficient, both in terms of inadequate consumption smoothing and misallocation of labor across occupations. SDI addresses both of these. It directly provides smoother consumption in risky occupations which indirectly serves to incentivize workers to choose riskier occupations. The next proposition 
shows that a marginal introduction of SDI improves both welfare and productive efficiency. It shifts labor to a more efficient allocation and provides smoother consumption across states.

Proposition 3.5 (There exists a strictly positive welfare maximizing level of insurance that is a Pareto improvement over no insurance.). Let $E U(j, \tau)$ be the expected utility $E_{j}\left[U\left(c_{1}^{*}(j), c_{n}^{*}(j), c_{d}^{*}(j)\right)\right](\tau)$ from the allocation in competitive equilibrium $\left\{c_{1}^{*}(j), c_{n}^{*}(j), c_{d}^{*}(j), a^{*}(j), n^{*}(j), \ell^{*}(j)\right\}$ satisfying Definition 3.1 for occupation-independent benefit rate $b$ funded by occupation-independent tax rate $\tau$. Then, $\exists \tau^{*}>0$ such that

$$
\frac{\partial \int \ell(j) E U\left(j, \tau^{*}\right) d j}{\partial \tau}=0
$$

and

$$
E_{j}\left[U\left(c_{1}^{*}(j), c_{n}^{*}(j), c_{d}^{*}(j)\right)\right]\left(\tau^{*}\right)>E_{j}\left[U\left(c_{1}^{*}(j), c_{n}^{*}(j), c_{d}^{*}(j)\right)\right](0) \quad \forall j
$$

Proof. See Appendix B.5.

A key insight is that the redistribution of workers across occupations under social insurance increases the wage in the risk-free occupation. This is a necessary condition for SDI to provide a Pareto improvement, regardless of specific parametrizations. Because workers are free to move across occupations, their welfare must equalize. In particular, there must be welfare gains in the risk-free occupation that does not benefit from a transfer in a disabled state. This is possible because each dollar of benefits offsets the wage premium required in risky occupations for self-insurance by more than one-to-one.

Welfare gains from expanding the SDI system are not without bounds. As the system becomes larger, it serves as an inefficient transfer to individuals in risky occupations. At the extreme of $100 \%$ taxation, the SDI system is clearly is clear suboptimal as every worker will choose the occupation that makes claiming disability insurance most likely. The following corollary shows that there exists and optimal SDI system between zero and this extreme. 
The welfare maximizing level of social insurance does not maximize output if occupation specific benefits do not exist. This is because a system that simultaneously maximizes output and welfare requires consumption to be perfectly smoothed across states in each occupation. In such a system there is no longer any risk in any occupation, workers then reallocate to equalize the marginal product across occupations, which is the efficient allocation. Without occupation specific benefits, the policy maker simply does not have sufficient policy tools to maintain indifference across occupations at the output maximizing allocation.

Proposition 3.6 (The welfare maximizing level of social insurance with occupation independent taxes and benefits does not maximize output.). Let $n^{c m}(j)$ characterize the efficient (output maximizing) allocation. $\operatorname{Let}^{r p}(j), w^{r p}(j) ; \tau, b$ be the constrained optimal planner allocation (maximizes welfare in competitive equilibrium given limited policy tools). Then $y^{r p}<y^{c m}$ and, in particular $\int_{j=0}^{t} n^{r p}(j) d j<\int_{j=0}^{k} n^{c m}(j)$ for all $k \in[0, J]$.

Proof. See Appendix B.7.

As a Corollary to Proposition 3.7, we show the first best, planner's allocation can be achieved if occupation specific benefits are available.

Corollary 3.7 (The first-best planner allocation can be achieved with a lump-sum or proportional tax and occupation-specific benefits.). Let $\mathcal{A}^{*}(\hat{b}, \hat{\tau})=$ $\left\{c_{1}^{*}(j), c_{n}^{*}(j), c_{d}^{*}(j), a^{*}(j), n^{*}(j), \ell^{*}(j)\right\}$ satisfying Definition 3.1 given arbitrary occupation-specific benefits $\hat{b}=\left\{b_{j}\right\}_{j}$ funded by occupation-specific taxes $\widehat{\tau}=$ $\left\{\tau_{j}\right\}_{j}$. Let $\mathcal{A}^{c m}=\left\{c_{1}^{c m}(j), c_{n}^{c m}(j), c_{d}^{c m}(j), a^{c m}(j), n^{c m}(j), \ell^{c m}(j)\right\}$ define the first-best planner's allocation. Then, $\exists \mathbf{b}, \tau$ such that $\mathcal{A}^{*}(\widehat{b}, \widehat{\tau})=\mathcal{A}^{c m}$

Proof. See Appendix B.7

Figure 4 shows features of welfare and output gains from the occupation independent tax and transfer social insurance system described in the propositions above. First, starting with no social insurance, the welfare and output 
gains are always positive. Second, there exists a unique output-maximizing tax level and a unique welfare-maximizing tax level. The welfare maximizing tax level does not coincide with the output maximizing tax level. For a range of parametrizations ( $\sigma=3$ shown), the welfare-maximizing system puts more labor in risky occupations than the output-maximizing (efficient) allocation. This means that at the output-maximizing allocation, there are greater gains from providing higher benefits for consumption smoothing than the output loss incurred as these benefits move more workers into risky occupations than is efficient.

[Figure 4 about here.]

In our model with homogeneous workers, SDI increases ex ante welfare uniformly because of indifference across occupations. Ex post, we can see more welfare changes relative to no SDI for workers in each state: disabled and non-disabled. To understand these welfare effects, consider an old generation who entered the labor market without any SDI program and hold fixed their asset choices. We then give them the wages, taxes, and benefits of various sized SDI programs. The welfare gains for this experiment, across occupations and disability outcome, are shown in Figure 5.

[Figure 5 about here.]

The first panel in Figure 5 shows that disability benefits improve the welfare of workers that become disabled. The second panel shows workers with low disability risk benefit even if they do not become disabled. This is because they earn higher wages after reallocation. Similarly, workers with high disability risk are actually worse off if they do not become disabled. This is because SDI causes more workers to enter the high risk occupations and lowers wages for workers in these occupations, but they gain in an ex ante sense due to risk sharing. The final panel shows the total expected benefit for workers in the second period of life. 


\section{Robustness and Extensions}

In this section we show the main results of the baseline model, that the introduction of SDI increases welfare through each channel of risk sharing and occupational reallocation, are robust to considerations specific to disability risk. We consider three potential extensions: heterogeneity in patience, costly disability, and non-verifiable disability status. Then, we show how public, social disability insurance dominates private contracts within our framework.

\subsection{Heterogeneity and Sorting}

A main concern in our empirical work was to control for the sorting of individual's into occupations based on fixed, ex-ante heterogeneity. There, we were concerned such a fixed trait could affect occupation choice and health. Here we investigate within our model how such a fixed trait could affect how individuals sort across occupations and how they value disability insurance. For exposition purposes, we consider heterogeneity in discount factor, $\beta$ and a two-type case, $\beta^{H}=1$ and $0<\beta^{L}=\beta<1$. Our results are general to $N$ types and adaptable to other forms of ex ante heterogeneity. Supporting proofs are in Appendix Section B.8.

Proposition 4.1 shows our assumptions on heterogeneity provide monotone occupational sorting in the form of a cut-off rule. ${ }^{4}$ Patient, high-beta types choose to occupy all of the less-risky occupations below an endogenous threshold $\bar{j}(\tau)$. Impatient low-beta types occupy the remaining riskier occupations.

\footnotetext{
${ }^{4}$ Sorting is also consistent with findings in our empirical work where the IV-implied effects were greater than OLS, which suggests sorting into high risk occupations. Our IV focused especially on tolerance to physical demands, but our conclusions from preference heterogeneity mostly extend to heterogeneity in health-sensitivity, though the model is less tractable.
} 
Proposition 4.1 (Monotone Occupational Sorting). There exists a unique $\bar{j}$ such that any occupation with $j<\bar{j}$ employs only high-beta types and any occupation with $j>\bar{j}$ employs only low-beta types.

Proof. See Appendix B.8.

Proposition 4.2 shows that the marginal introduction of SDI increases welfare for both types through the same channels, risk sharing and occupational allocation. The first-best planner's labor allocation is the same as without preference heterogeneity: the allocation of workers to occupations is chosen to achieve productive efficiency. This efficient distribution of workers first-order stochastically dominates the distribution in the competitive allocation.A marginal introduction of SDI improves productive efficiency. It moves the labor allocation towards more risky occupations, both within beta-types and by moving the threshold $\bar{j}(\tau)$ of occupations held by high-beta types upwards.

Proposition 4.2 (Social Insurance is Welfare Improving (on the margin) for all Beta-Types). Let $E U^{i}(\tau)$ be the expected utility of a type $\beta^{i} \in\left\{\beta^{h}, \beta^{\ell}\right\}$ agent from the competitive equilibrium in the case of (i) a continuum of occupations; (ii) two types of agents with different discount factors; (ii) and proportional social insurance $\left(\tau \geq 0, b_{j}=b w_{j}\right.$, and $\sum_{j}\left(2-\theta_{j}\right) \ell_{j} \tau w_{j}=$ $\left.\sum_{j} \theta_{j} \ell_{j} b w_{j}\right)$. Then:

$$
E U^{\prime i}(0)>0 \quad \forall i \in\{\ell, h\}
$$

Proof. See Appendix B.11.

While all workers' welfare improves, an interesting result is that workers in risky occupations can have lower welfare gains from introducing SDI than workers in less risky occupations. As in Schulhofer-Wohl (2011), some agents value insurance less than others. These low-beta types also gain the least from the second channel, occupational reallocation. As the threshold $\bar{j}(\tau)$ increases the patient workers move into the riskier jobs previously held by 
low-beta types thus lowering their wage premium. Conversely, workers with low disability risk value the insurance benefits of SDI more than individuals with high risk and see an increase in their relative wage. This is a qualitative distinction from models of SDI that treat disability risk as common across individuals.

\subsection{Costly Disability}

Disability is different from other types of income risk in that the decline in income associated with disability occurs in conjunction with an increase in expenditure costs. These costs range from direct medical costs to increased expenditure on goods and services that an individual can no longer produce themselves. We introduce such costs into the model here, with technical details in Appendix Section B.9. Let preferences over consumption in the disabled state be represented by the utility function $u^{d}$. We assume:

(i) $u_{d}(c(1+\chi))=u(c) \quad ; \quad(i i) \quad u_{d}^{\prime}(c(1+\chi)) \propto u^{\prime}(c) \quad ; \quad \chi \geq 0, \quad \forall c>0$.

That is the cost of disability is a constant proportion of consumption $(1+\chi)$ required to regain the utility of the non-disabled. This is analogous to the preferences used in Low et al. (2015). Costs of disability have constant marginal relationships with the level of consumption and we can isolate concerns with productive efficiency and risk-sharing separately.

With this modification, the social planner's problem becomes:

$$
\begin{gathered}
\max _{\ell(j), c_{d}(j), c_{n}(j), c_{1}(j)} \int_{j}\left[u\left(c_{1}(j)\right)+\theta(j) u^{d}\left(c_{d}(j)\right)+(1-\theta(j)) u\left(c_{n}(j)\right)\right] \ell(j) d j \\
\text { s.t. } \int_{j} \ell(j)\left[c_{1}(j)+\theta(j) c_{d}(j)+(1-\theta(j)) c_{n}(j)\right] d j \leq\left(\int_{j}(2-\theta(j))^{\gamma} \ell(j)^{\gamma} d j\right)^{\frac{1}{\gamma}}, \quad \int_{j} \ell(j) d j=1
\end{gathered}
$$

The solution to this problem delivers two main results. First, the allocation of consumption equalizes marginal utilities across disabled and non-disabled 
individuals: $c=c_{1}=c_{n}=(1+\chi) c_{d}$. Second, the labor in occupation $k$ relative to occupation $j$ at the optimal allocation of labor is:

$$
\frac{\ell(k)}{\ell(j)}=\left(\frac{(2-\theta(k))}{(2-\theta(j)}\right)^{\frac{\gamma}{1-\gamma}}\left(\frac{(2+\chi \theta(k))}{(2+\chi \theta(j))}\right)^{\frac{1}{1-\gamma}}
$$

With $\chi=0$, this is equivalent to the base case without a cost of disability. We see the disability cost, $\chi>0$ reduces labor in the high-risk occupation. The relative marginal-product is now $\frac{2-\theta(j)}{2+\chi \theta(j)} / \frac{2-\theta(k)}{2+\chi \theta(k)}: \chi>0$ drives a wedge between the relative marginal product of labor between two occupations of different risk. Increasing this cost $\chi$ increases the difference in the marginal product of labor between two occupations for all values of $\gamma$, even strong complements.

In a sense, this is a second cost of disability in our model. The first cost was the "human capital" cost of fewer workers given by the fraction $\theta(j)$ who cannot work in the second period. The key difference is that the impact of the human capital cost on the planner's allocation depends on the elasticity of substitution across occupations. For $\gamma>0$, the occupations are gross complements and the planner actually puts more labor in risky occupations relative to a safe ones. The qualitative impact of the utility costs captured by $\chi$ does not depend on this elasticity; these costs always decrease relative labor in risky occupations. However, the magnitude of the effect is increasing in the substitutability of occupations.

Proposition 4.3 shows our main result holds in this environment: the introduction of SDI improves welfare through both the risk sharing channel and channel of labor reallocation that increases aggregate productivity. However, this result does not hold for arbitrary $\operatorname{costs} \chi$. A sufficient restriction is that the disability cost must not exceed the elasticity of the benefit replacement rate $b$ to the tax $\tau$. Such an assumption ensures that a cost of $\tau c$ in the non-disabled state is offset by a benefit of $\frac{b}{1+\chi} c$ in the disabled state.

Proposition 4.3 (Social Insurance is Welfare Improving (on the margin)). Let $E U(\tau)$ be the expected utility of an agent from the competitive equilibrium 
in the case of (i) a continuum of occupations; (ii) costly disability; and (iii) and proportional social insurance $\left(\tau \geq 0, b_{j}=b w_{j}\right.$, and $\sum_{j}\left(2-\theta_{j}\right) \ell_{j} \tau w_{j}=$ $\left.\sum_{j} \theta_{j} \ell_{j} b w_{j}\right)$. Then:

$$
\frac{d E U(\tau)}{d \tau} \|_{\tau=0}>0
$$

Proof. See Appendix B.15.

\subsection{Non-verifiable Disability Risk and Status}

Here, we consider optimal SDI when neither ex-ante disability risk nor realized disability status is observable. In our baseline model, we have already restricted SDI not to condition on occupation, therefore the a scheme is robust to non-verifiable ex ante disability risk, $\theta(j)$. For the latter, truthtelling about current disability status holds below a bound on insurance. In other words, if the replacement rate $b$ is less than $1-\tau$, given that benefits and taxes are both proportional to wages a non-disabled agent clearly gains from working, and therefore will not misreport her status. Proposition 4.4 makes explicit this result in our two period economy. However, it is a concern for the quantitative economy in Section 5 when there is a large population of young workers relative to older workers at risk of disability.

Proposition 4.4 (The Optimal SDI Policy is Robust to Non-Verifiable Disability Status). Let $\left\{c_{1}^{*}\left(j, \tau^{*}\right), c_{n}^{*}\left(j, \tau^{*}\right), c_{d}^{*}\left(j, \tau^{*}\right), a^{*}\left(j, \tau^{*}\right), w\left(j, \tau^{*}\right)\right\}_{j=1}^{J}$ satisfy the definition of a competitive equilibrium at the optimal SDI policy $(\tau, b=b(\tau))$. Then, the expected utility of reporting disability status truthfully, $E U(j, \tau)$, is greater than any time-consistent deviation:

$$
\begin{gathered}
E U(j, \tau)=\max _{a} u((1-\tau) w(j, \tau)-a)+\theta(j) u(b w(j, \tau)+a)+(1-\theta(j)) u((1-\tau) w(j, \tau)+a) \\
\geq \max _{a} u((1-\tau) w(j, \tau)-a)+\theta(j) u(b w(j, \tau)+a) \\
\quad+(1-\theta(j)) \max \{u((1-\tau) w(j, \tau)+a), u(b w(j, \tau)+a)\}
\end{gathered}
$$


Proof. See Appendix B.16.

\subsection{Private Contracts}

In the US, private disability insurance often has relatively small payouts and is not universally offered. Because of this, Golosov and Tsyvinski (2006) do not consider private contracts. Here, we will show numerically how adverse selection hinders private disability insurance, providing a rationale for social insurance. Private insurance contracts induce too much demand from workers in high-risk occupations, sullying the risk pool. Private insurance contracts therefore earn negative profits, because at any price and benefit that would be feasible with compulsory SDI, low-risk agents opt not to buy. The market breaks down in a way typical of averse selection.

The crucial constraint for private insurance is that, like social insurance, it cannot condition on the worker's occupational risk level, $\theta(j) .{ }^{5}$ But, whereas social insurance can dictate the amount of insurance any agent takes, private insurance cannot. With social insurance, workers can only increase their exposure to the insurance by increasing their exposure to disability risk, which we showed was actually optimal to an extent (Proposition 3.5). Instead, with private there is over-subscription into these contracts from the workers already in the risky occupations. On the other hand, if occupational risk-level were verifiable and private insurance contracts on it, these can dominate social insurance. We do not explore that here further because relaxing a constraint for the private insurers makes the comparison uneven.

To illustrate how private disability insurance breaks down, we consider our baseline economy, fixing labor at its efficient allocation and then introducing private disability contracts. Given an occupation $j$ and insurance

\footnotetext{
${ }^{5}$ If either private or social insurance can verify disability risk level (given by occupation) then they can achieve the first best allocation. This is the case with complete markets (Section B.1, Equation B.16). For comparison's sake, we keep the same information restrictions on both social and private insurance.
} 
price $p$, agents solve for optimal disability insurance $g$ :

$$
\max _{g, a} u\left(w^{C M}(j)-p w^{C M}(j) g-a\right)+\theta(j) u\left(b w^{C M}(j) g+a\right)+(1-\theta(j)) u\left(w^{C M}(j)+a\right)
$$

Then the benefits $b$ are set to satisfy the insurers budget constraint if they could enforce uniform participation, the best-case scenario.

$$
\int_{j} \theta(j) p w^{C M}(j)-(2-\theta(j)) b w^{C M}(j) d j=0
$$

At any price, the private insurance contracts incur negative profits, as shown in the left pane of Figure 6 because those in high-risk occupations over subscribe, as shown in the right pane. The insurer could increase $p$ or reduce $b$, but both of these serve only to worsen the selection problem. Figure 6 shows how increases in $p$ lead to increases in the average $\theta(j)$ among the insured population and the effect is analogous if the insurer dropped $b$. For private contracts with positive $p$ and $b$, profits are negative. Social disability insurance can mandate broad participation across risk levels, but private disability insurance fails here because of averse selection: the workers who chose to participate most are the worst risks.

[Figure 6 about here.]

\section{Quantitative Evaluation}

We now provide a simple quantitative model to explore the relative magnitudes of reallocation and risk-sharing within the policy region of the US and explore policy counterfactuals.

\subsection{Calibration}

For this exercise, we maintain the two-generation overlapping generations structure of the theory section but modify the duration of the first period to 
30 years (ages 25-55) and the second period to 10 years (ages 55-65). ${ }^{6}$ We consider only two occupations, guided by the natural break in occupational risks seen in Figure 1. We target statistics we documented associated with this break: the disability risk in the high-risk occupation is twice that of the low-risk occupation $\left(\pi^{h}=2 \pi^{\ell}\right)$ and $17 \%$ of the young choose the highrisk occupation. We choose the low-risk occupation's disability hazard to be $\pi^{\ell}=0.13$. This provides a disability beneficiary share of the population equal to the (demographic adjusted) average from 1985-2016: 3.9\%.7

We give the social disability system a proportional tax and replacement rate and the same tools to our constrained optimal planner. The baseline replacement rate to 40\% (see Duggan and Autor (2006)) and then solve for the implied balanced-budget tax.

Preferences follow Low et al. (2015): $u(c)=\frac{(c)^{1-\sigma}-1}{1-\sigma}$ for the non-disabled and $u_{d}(c)=\frac{(c * \exp (\eta))^{1-\sigma}-1}{1-\sigma}$ for the disabled. We use their calibrated parameters: $\sigma=1.5$ following the literature, and $\eta=-0.448$ following their estimation using micro-data on consumption. The production technology uses low-disability-risk labor $n_{\ell}$ and high-risk labor $n_{h}: Y=Q\left(\alpha n_{\ell}^{\gamma}+(1-\alpha) n_{h}^{\gamma}\right)^{1 / \gamma}$. We compute results for an elasticity of substitution ranging from $\frac{1}{3}$ to $5 .^{8}$ For each $\gamma$, we recalibrate $\alpha$ to match $17 \%$ of workers choosing the high-risk occupation (to match the statistic we documented in the HRS data) and set $Q$ to normalize output to 1.

\footnotetext{
${ }^{6}$ This break is motivated by the fact that $71 \%$ of disabled beneficiaries were 55 years of age or older in 2016 (Authors' calculations from data released with Administration (2017).

${ }^{7}$ Authors' calculation following Michaud and Wiczer (2017). The choice of $\pi^{\ell}=0.13$ is not far from the analogous ADL risk of $8 \%$ in the low-risk cluster of occupations.

${ }^{8}$ See Katz and Autor (1999) for a literature review on the substitutability of differentiated types of labor, broadly defined. Most studies place this number between 1 and 2 . We provide results for a wider range both because the dispersion of estimates is high and to better understand the quantitative properties of mechanism we are studying.
} 


\subsection{Results}

Using the calibrated production, preference, and occupational risk parameters, we calculate the equilibrium under two different insurance policies: the constrained optimal equilibrium and an equilibrium with "actuarially fair" insurance, defined by benefits that cover the expected income loss for the average worker. Table 3 presents aggregate statistics for each of these two counterfactual policy regimes as well as the baseline calibrated to the US system. Relative to the actuarially fair system, the constrained optimal SDI system is a substantial improvement in welfare, $2.3 \%$ in consumption equivalent terms. Further, the constrained optimal policy increases welfare over the baseline by $1.5 \%$. This necessitates large changes in policy including a three-fold increase in the replacement rate and a doubling of the tax rate. This expanse is driven by the Low et al. (2015) calibration of the increased marginal utility of consumption for the disabled.

[Table 3 about here.]

To help evaluate the channel of productive efficiency, Figure 7 shows the labor allocation under each policy while changing the elasticity of substitution. The baseline target of $17 \%$ of workers in the risky occupation is fewer than the output-maximizing allocation. The constrained optimal allocation moves beyond productive efficiency, a general result proved in Proposition 3.6. However, there are negligible output effects when comparing the baseline and constrained optimal allocation. This is an artifact of the calibration of the weight $\alpha$ in the production technology required to match $17 \%$ of workers in the risky occupation. For very low elasticity of substitution, the calibration implies a very low output weight on high-risk occupations and therefore little effect, whereas for very high substitutability again output effects are small because workers are essentially fungible. Thus, welfare gains of the constrained optimal allocation compared to the baseline come primarily from increased risk-sharing with little output losses from moving beyond productive efficiency. 
[Figure 7 about here.]

\section{Conclusion}

In this paper, we have explored how occupational choice shapes disability risk and policy. We first documented the importance of lifetime occupational exposure to differences in disability risk. We then embedded this idea-occupational choices imply different levels of disability risk - into an equilibrium model with incomplete asset markets and imperfectly substitutable occupations. Here, incomplete markets for disability risk lead to both imperfect risk-sharing and an inefficient allocation of labor across occupations. This leaves room at the margin for a welfare-improving disability insurance, which improves consumption smoothing and reallocates workers to increase output. This latter point resembles moral hazard: insuring risky occupations encourages more risk-taking, but at the margin this is efficient.

We provided quantitative insights from a two-occupation model calibrated to resemble the United States. We found that the welfare-maximizing SDI provides welfare gains that are equal to a $2.3 \%$ increase in consumption in a world with actuarially fair insurance alone and $1.5 \%$ when compared to the current policy regime. However, these gains come primarily through additional risk sharing, not productive efficiency.

For future research, our results on private insurance indicated potentially new avenues. Data on private disability insurance is not inconsistent, but is more complicated than our model admits: Private disability insurance is very rarely sold directly to individuals, in 2015 there were about a half-million in the US (Isenberg (2016)). Employer-provided disability insurance is instead

much more common, covering about $\frac{1}{3}$ of the workforce. This coverage, however, is essentially rationed and occupation is a good indicator of access. $98 \%$ of workers enroll if DI is offered by their employers, but offers are very hetero- 
geneous across occupations (Groshen and Perez (2015)). ${ }^{9}$ Management and professional occupations, relatively low risk, are offered long-term disability insurance $50 \%$ of the time, while high risk work, production occupations and construction or extraction occupations are only offered it $28 \%$ and $20 \%$ of the time, respectively. Employer provision of disability insurance seems to be one way private insurance can condition on $\theta(j)$, by utilizing employers' additional information and then rationing insurance.

\footnotetext{
${ }^{9}$ This large heterogeneity across occupations in access to disability insurance is, a useful validation of our empirical work, showing evidence that private markets treat occupations differently in what private disability insurance exists.
} 


\section{Online, not-for-publication appendix}

\section{A. Empirical appendix}

In this Appendix, we include details about our instrumental variables approach using $\mathrm{O}^{*} \mathrm{NET}$ measures and robustness using an alternative disability definition.

\section{A.1. Data construction}

Health and Retirement Study sample. The HRS surveys households whose head is older than 50 years of age and also includes data on their "spouses," potentially an unmarried cohabitant. The panel is collected biannually from 1992 to 2010. The individual listed as a spouse may change through the survey waves and we drop these observations. After excluding observations for non-responses, we have between 16,128 and 21,623 observations per wave with a total of 184,541 .

In each wave, individuals report difficulties they have across many ADLs. These include walking across a room, getting dressed, bathing, and getting in and out of bed. We record an indicator once a worker begins to continuously report a difficulty with any ADL. ${ }^{10}$

We associate individuals with their longest-held occupation. This is a retrospective question in each wave. Because "longest-held" is repeatedly

\footnotetext{
${ }^{10} \mathrm{We}$ also experimented with a metric over the number and severity of the difficulties, a kind of frailty index. Results are all robust to this definition and are available upon request.
} 
asked each wave, we take the occupation with the longest tenure. ${ }^{11}$ The longest-held occupation has a median tenure of 19 years and the bottom quartile is 11 years.

\section{A.2. Instrumental Variables Stage One}

Table 4 presents the first stage regression results for the endogenous variable $H_{j}$, health requirements in occupation $j$. Each non-physical requirement is standardized to have a mean 0 and standard deviation of 1 . The requirements are defined by the first 3 orthogonal principal components of $\mathrm{O}^{*} \mathrm{NET}$ descriptors excluding physical requirements.

[Table 4 about here.]

[Table 5 about here.]

\section{A.3. Robustness: Linear Probability Model}

In this section we replicate Table 2 but with a linear probability model (LPM) rather than Probit. While the OLS estimator in an LPM has obvious drawbacks, i.e. predicted probabilities may go outside of $(0,1)$, it is somewhat less reliant on distributional assumptions than probit. The coefficients in the LPM and IV-LPM are quantitatively quite close to those in the Probit model, as we show in Table A.3

[Table 6 about here.]

\footnotetext{
${ }^{11}$ The longest-held occupation may change from wave to wave because the individual's tenure in the current occupation overtakes the prior longest-held occupation or because of coding error. The latter occurs $4 \%$ of the time in the first wave, but then less than $1 \%$ of the time. We overwrite these coding errors.
} 


\section{B. Theoretical Results and Proofs}

\section{B.1. Setting up the tax-free economy}

We first define the complete and incomplete markets versions of our taxfree economy $(\tau=b=0)$. In both cases, firms operate in spot markets (no long-term contracting). The representative firm solves

$$
\max _{\{n(j)\}}\left(\int_{j=0}^{J} n(j)^{\gamma}\right)^{1 / \gamma} d j-\int_{j=0}^{J} w(j) n(j) d j
$$

In equilibrium all non-disabled workers are hired: $n(j)=(2-\theta(j)) \ell(j)$. From this problem, the first-order condition (FOC) is

$$
w(j)=\left(\frac{y}{n(j)}\right)^{1-\gamma}
$$

And for any occupations $j, k$

$$
\frac{w(j)}{w(k)}=\left(\frac{n(j)}{n(k)}\right)^{\gamma-1}
$$

Complete Markets. In this section, we identify the endogenous variables of the competitive equilibrium with the superscript $\mathrm{cm}$. We begin by setting up the households' problem with the complete set of Arrow securities along with a backyard storage technology earning return $R_{0}$. It is trivial to show directly that these allocations correspond to those in the social planner's problem because the absence of discounting combined with a backyard storage technology solves typical incomplete markets inefficiencies associated with OLG economies. With the full set of assets, both welfare theorems hold and the efficient allocation, $\left\{c_{1}^{c m}(j), c_{d}^{c m}(j), c_{n}^{c m}(j), \ell^{c m}(j)\right\}$, is also unique.Their possible states are $s=\{1, d, n\}$ for period 1, 2 as disabled, and 2 as nondisabled; and $j$ for the occupation they choose. The full set of Arrow securities, $\left\{a_{d}(j), a_{n}(j)\right\}$ spans states $j, s$ : 


$$
\begin{aligned}
\max _{\left\{c_{s}(j), \ell(j), a_{n}(j), a_{d}(j)\right\}} & \int_{j=0}^{J}\left\{u\left(c_{1}(j)\right)+\theta(j) u\left(c_{d}(j)\right)+(1-\theta(j)) u\left(c_{n}(j)\right)\right\} \ell(j) d j \\
c_{1}(j) & \leq w(j)-a(j)-\left(\int_{k=0}^{J} a_{n}(k)+a_{d}(k)\right) d k \\
c_{n}(j) & \leq w(j)+R_{0} a(j)+\int_{k} R_{n}(k) a_{n}(k) d k \\
c_{d}(j) & \leq R_{0} a(j) \int_{k} R_{d}(k) a_{d}(k) d k \\
1 & \geq \int_{j} \ell(j) d j
\end{aligned}
$$

The solution to this problem sets each interest rate $R_{n}(j)=\frac{1}{1-\theta(j)}$, $R_{d}(j)=\frac{1}{\theta(j)}$ and the saving policies are $a_{n}^{c m}(j)=\frac{-\theta(j)}{2}(1-\theta(j)) w^{c m}(j)$; and $a_{d}^{c m}(j)=\frac{2-\theta(j)}{2} \theta(j) w^{c m}(j)$ for $j$ such that $\ell(j)=1$ and $a_{s}^{c m}(k)=0 \forall k \neq j$. This implies consumption is smoothed across time and states

$$
c_{1}^{c m}(j)=c_{n}^{c m}(j)=c_{d}^{c m}(j)=\frac{2-\theta(j)}{2} w^{c m}(j)
$$

The occupation choice $\ell(j)$ requires expected earnings to be equalized across occupations $j, k$ :

$$
w^{c m}(j)(2-\theta(j))=w^{c m}(k)(2-\theta(k))
$$

Combining the wage condition with consumption gives

$$
w^{c m}(j)=\frac{2 c}{2-\theta(j)}
$$

where $c$ is the consumption level of every individual. From the firms' side, Equation B.1, and substituting market clearing that $y=2 c$, and $\ell^{c m}(j)(2-$ $\theta(j))=n(j)$, we also have

$$
w^{c m}(j)=\left(\frac{2 c}{(2-\theta(j)) \ell^{c m}(j)}\right)^{1-\gamma}=\frac{2 c}{2-\theta(j)}
$$


Solving for $\ell^{c m}(j)$ gives

$$
\ell^{c m}(j)=\left(\frac{2 c}{2-\theta(j)}\right)^{\frac{\gamma}{\gamma-1}}
$$

Now, we can use the market clearing condition that $\int_{j} \ell^{c m}(j) d j=1$ to solve for $c$, and hence $\ell^{c m}(j)$. That is, $1=\left(\int_{j} \frac{2 c}{2-\theta(j)} d j\right)^{\frac{\gamma}{\gamma-1}}$, which, solving for $c$, implies that

$$
c=\frac{1}{2}\left(\int_{j=0}^{J}(2-\theta(j))^{\frac{\gamma}{1-\gamma}} d j\right)^{\frac{1-\gamma}{\gamma}}
$$

and then plugging that in:

$$
\ell(j)=(2-\theta(j))^{\frac{\gamma}{1-\gamma}}\left[\int_{k=0}^{J}(2-\theta(k))^{\frac{\gamma}{1-\gamma}} d k\right]^{-1}
$$

Social Planner. Notice that the complete markets allocation will yield the same labor allocation as the social planner and hence the same output. Because consumption is also equal across all states and generations, the social planner and complete markets allocations are the same and the complete markets allocation is efficient.

The social planner solves

$$
\begin{aligned}
\max _{\ell(j), c(j)} & \int_{j} \lambda(j) 2 u(c(j)) \ell(j) \text { s.t. } \\
\left(\int_{j}((2-\theta(j)) \ell(j))^{\gamma} d j\right)^{1 / \gamma} & \geq \int_{j} l(j) 2 c(j) d j \\
\int_{j} \ell(j) d j & \leq 1
\end{aligned}
$$

where we have already plugged in the obvious result that the social planner provides households with perfectly smooth consumption, setting it to be equal in both periods and all states. $\lambda(j)$ defines arbitrary Pareto weights.

The FOC on labor implies that

$$
\left(\frac{y^{c m}}{\ell^{c m}(j)(2-\theta(j))}\right)^{1-\gamma}(2-\theta(j))=\left(\frac{y^{c m}}{\ell^{c m}(k)(2-\theta(k))}\right)^{1-\gamma}(2-\theta(k))
$$


for any $j, k$ where $y^{c m}=\left(\int_{j}\left((2-\theta(j)) \ell^{c m}(j)\right)^{\gamma}\right)^{1 / \gamma}$. Then, express all $\ell^{c m}(k)$ in terms of $\ell^{c m}(j)$ and we can use the condition on labor, Equation B.14 to sum up:

$$
1=\int_{k}\left(\frac{\ell(j)^{c m} \gamma-1(2-\theta(j))^{\gamma}}{(2-\theta(k))^{\gamma}} d k\right)^{\frac{1}{\gamma-1}}
$$

Solving gives

$$
\ell^{c m}(j)=(2-\theta(j))^{\frac{\gamma}{1-\gamma}}\left[\int_{k}(2-\theta(k))^{\frac{\gamma}{1-\gamma}} d k\right]^{-1}
$$

Note that this is the same outcome as our complete markets setup and hence output is the same in both worlds. Because consumption is equal for all people in all states in both frameworks, we have shown that the allocations are equivalent.

Incomplete Markets. With only one asset, households solve

$$
\begin{aligned}
& \max _{j, a} u(w(j)-a)+\theta u(a)+(1-\theta(j)) u(w(j)+a) \\
& \{a\}: u^{\prime}\left(w^{*}(j)-a^{*}(j)\right)=\theta u^{\prime}\left(a^{*}(j)\right)+(1-\theta(j)) u^{\prime}\left(w^{*}(j)+a^{*}(j)\right) \\
& \{j\}: u\left(w^{*}(j)-a^{*}(j)\right)+\theta(j) u\left(a^{*}(j)\right)+(1-\theta(j)) u\left(w^{*}(j)+a^{*}(j)\right) \\
& \quad=u\left(w^{*}(k)-a^{*}(k)\right)+\theta(k) u\left(a^{*}(k)\right)+(1-\theta(k)) u\left(w^{*}(k)+a^{*}(k)\right)
\end{aligned}
$$

The FOC on occupation choice that households are indifferent between choosing any risk level $\theta(j), \theta(k)$. We have denoted the optimal savings policy of an agent choosing $j$ as $a^{*}(j)$, which is distinct from the complete markets case where we indexed potential $a_{s}(k)$ by the level of risk assumed and the occupation that would buy them.

Consider the case $\theta(k)=0$, then the two first-order-conditions imply

$$
u\left(w^{*}(j)-a^{*}(j)\right)+\theta(j) u\left(a^{*}(j)\right)+(1-\theta(j)) u\left(w^{*}(j)+a^{*}(j)\right)=2 u\left(w^{*}(k)\right)
$$


Note also that the Inada condition on utility guarantees that for $j>0$, $\theta(j)>0$ implies $a^{*}(j)>0$ otherwise in the disabled state marginal utility is not finite.

\section{B.2. Inefficiency of the incomplete markets equilibrium}

Proof of 3.3 . (reprinted here): Let $\left\{c_{1}^{*}(j), c_{n}^{*}(j), c_{d}^{*}(j), a^{*}(j), n^{*}(j), \ell^{*}(j)\right\}$ satisfy Definition 3.1 for the case $b=\tau=0$. There exists an alternative feasible allocation $\left\{\hat{c}_{1}(j), \hat{c}_{d}(j), \hat{c}_{n}(j), \hat{\ell}(j)\right\}$ such that

$$
\begin{aligned}
& E_{j}\left[U\left(\hat{c}_{1}(j), \hat{c}_{n}(j), \hat{c}_{d}(j)\right)\right] \geq E_{j}\left[U\left(c_{1}^{*}(j), c_{n}^{*}(j), c_{d}^{*}(j)\right)\right] \forall j \in[0, J] \\
\exists k \text { s.t. } & E_{k}\left[U\left(\hat{c}_{1}(k), \hat{c}_{n}(k), \hat{c}_{d}(k)\right)\right]>E_{k}\left[U\left(c_{1}^{*}(k), c_{n}^{*}(k), c_{d}^{*}(k)\right)\right]
\end{aligned}
$$

where $E_{s}$ conditions on disability probability $\theta_{s}$; and

$$
\int_{j} \hat{\ell}(j)\left(\hat{c}_{1}(j)+\theta(j) \hat{c}_{d}(j)+(1-\theta(j)) \hat{c}_{n}(j)\right) d j \leq\left(\int_{j}(\hat{\ell}(j)(2-\theta(j)))^{\gamma}\right)^{\frac{1}{\gamma}}
$$

Proof. It is easy to construct one such, Pareto dominating allocation to the incomplete markets competitive allocation. Define $\hat{\ell}(j)=\ell^{*}(j)$ and $\hat{w}(j)=$ $w^{*}(j)$ for all $j$, the same values as the competitive equilibrium. Next set $R_{d}(j)=\frac{1}{\theta(j)}, \hat{R}_{n}(j)=\frac{1}{1-\theta(j)} \quad \forall j$. Define the remaining allocations as the solution to agents' problem taking prices $\{\hat{w}(j)\}$ and $\left\{\hat{R}_{n}(j), \hat{R}_{d}(j)\right\}$ as given:

$\max _{c_{1}(j), c_{n}(j), c_{d}(j), a_{d}(j), a_{n}(j)} u\left(\hat{w}(j)-a_{d}(j)-a_{n}(j)\right)+\theta(j) u\left(a_{d}(j) \hat{R}_{d}(j)\right)+(1-\theta(j)) u\left(\hat{w}(j)+a_{n}(j) \hat{R}_{n}(j)\right)$

We get fully smooth consumption consumption, $\hat{c}_{1}(j)=\hat{c}_{n}(j)=\hat{c}_{d}(j)=$ $\frac{2-\theta(j)}{2} \hat{w}(j)$. This is clearly feasible, as we have only redistributed the same output among workers who choose occupation $j$. This is strictly preferred for each $j \in(0, J]$ because of strict concavity and for $j=0$, the workers in the risk-free occupation are indifferent. 


\section{B.3. Comparing the complete and incomplete markets allocations}

Lemma B.1. Compared to the incomplete markets allocation, the complete markets allocation has more mass in every risky occupation relative to the zero-risk occupation: $\forall j \in(0, J], \frac{\ell(j)}{\ell(0)}<\frac{\ell^{c m}(j)}{\ell^{c m}(0)}$.

Proof. To prove this, we use contradiction. Hence, we begin with the counter factual, ${ }^{12}$

$$
\exists j \text { s.t. } \frac{\ell(j)}{\ell(0)} \geq \frac{\ell^{c m}(j)}{\ell^{c m}(0)}
$$

We can then take this to the wage space using Equation B.2. First multiply both sides, $\frac{(2-\theta(j)) \ell(j)}{2 \ell(0)} \geq \frac{(2-\theta(j)) \ell^{c m}(j)}{2 \ell^{c m}(0)}$. Raising both the power $\gamma-1$ we get, subject to a parameter restriction that $\gamma<1$

$$
\left(\frac{(2-\theta(j)) \ell(j)}{2 \ell(0)}\right)^{\gamma-1} \leq\left(\frac{(2-\theta(j)) \ell^{c m}(j)}{2 \ell^{c m}(0)}\right)^{\gamma-1}
$$

This implies, by Equation B.2

$$
\frac{w(j)}{w(0)} \leq \frac{w^{c m}(j)}{w^{c m}(0)}
$$

Next, from the occupation choice indifference condition, workers must be indifferent between either occupation

$$
u\left(w(j)-a^{*}(j)\right)+\theta(j) u\left(a^{*}(j)\right)+(1-\theta(j))\left(w(j)+a^{*}(j)\right)=2 u(w(0))
$$

And, by Equation B.22 and monotonicity

$$
2 u(w(0)) \geq 2 u\left(\frac{w^{c m}(0)}{w^{c m}(j)} w(j)\right)
$$

Then, because $\frac{w^{c m}(0)}{w^{c m}(j)}=\frac{2-\theta(j)}{2}$ as shown in Equation B.8, we get that

$$
2 u\left(\frac{w^{c m}(0)}{w^{c m}(j)} w(j)\right)=2 u\left(w(j) \frac{2-\theta(j)}{2}\right)
$$

${ }^{12}$ It is trivial to show the case of $\theta(0)=0$ because $\frac{\ell(0)}{\ell(0)}=1=1=\frac{\ell^{c m}(0)}{\ell^{c m}(0)}$. Hence, we prove for cases in which $\theta(j) \in(0, J]$ 
where the right-hand side is the expected earnings from occupation $\theta$. Combining these,

$u\left(w(j)-a^{*}(j)\right)+\theta(j) u\left(a^{*}(j)\right)+(1-\theta(j))\left(w(j)+a^{*}(j)\right)=2 u(w(0)) \geq 2 u\left(w(j) \frac{2-\theta(j)}{2}\right)$

But this means that the incomplete markets, risky allocation is weakly preferred to the expected earnings, which violates Jensen's inequality with strictly concave preferences. Hence, our assumption in Equation B.21 could not be and we have established our Lemma B.1.

Corollary B.2. There is more mass at the risk-free occupation, where $\theta(0)=$ 0 , in the incomplete markets allocation with $\tau=0$ than the efficient allocation: $\ell^{*}(0)>\ell^{c m}(0)$

Proof. This is easy to see, using the fact that both $\ell^{*}, \ell^{c m}$ are densities and hence $\int_{j} \ell^{*}(j)=\int_{j} \ell^{c m}(j)=1$.

Taking inequality $\frac{\ell^{*}(j)}{\ell^{*}(0)}<\frac{\ell^{c m}(j)}{\ell^{c m}(0)} \forall j>0$ and integrating over $j$ we have

$$
\int_{j} \frac{\ell^{*}(j)}{\ell^{*}(0)} d j<\int_{j} \frac{\ell^{c m}(j)}{\ell^{c m}(0)} d j \Leftrightarrow \frac{\int \ell^{*}(j) d j}{\int \ell^{c m}(j) d j}<\frac{\ell^{*}(0)}{\ell^{c m}(0)}
$$

Then because $\int_{j} \ell^{*}(j) d j=\int_{j} \ell^{c m}(j)=1$ by definition, this gives us $1<\frac{\ell^{*}(0)}{\ell^{c m}(0)}$

\section{B.4. Inefficiency of competitive incomplete markets allocation}

Proof of 3.3. (reprinted here): Let $\left\{c_{1}^{c m}(j), c_{d}^{c m}(j), c_{n}^{c m}(j), \ell^{c m}(j)\right\}$ be the efficient, planner's allocation solving

$$
\begin{aligned}
\max _{\left\{c_{1}(j), c_{d}(j), c_{n}(j), \ell(j)\right\}} & \int_{j} \ell(j)\left(u\left(c_{1}(j)\right)+\theta(j) u\left(c_{d}(j)\right)+(1-\theta(j)) u\left(c_{n}(j)\right)\right) d j \text { s.t. } \\
\left(\int_{j}((2-\theta(j)) \ell(j))^{\gamma} d j\right)^{\frac{1}{\gamma}} & \geq \int_{j} \ell(j)\left(c_{1}(j)+\theta(j) c_{d}(j)+(1-\theta(j)) c_{n}(j)\right) d j \\
1 & \geq \int_{j} \ell(j) d j
\end{aligned}
$$


Then $\left\{c_{1}^{c m}(j), c_{d}^{c m}(j), c_{n}^{c m}(j), \ell^{c m}(j)\right\}$ strictly Pareto dominates the competitive equilibrium with zero taxes $\left\{c_{1}^{*}(j), c_{d}^{*}(j), c_{n}^{*}(j), \ell^{*}(j)\right\}$

Proof. First, note that both allocations satisfy feasibility because of the problems from which they are defined. That is

$$
\int_{j} \ell(j)\left(c_{1}(j)+\theta(j) c_{d}(j)+(1-\theta(j)) c_{n}(j)\right) d j \leq\left(\int_{j}(\ell(j)(2-\theta(j)))^{\gamma} d j\right)^{\frac{1}{\gamma}}
$$

for both $\left\{c_{1}^{c m}(j), c_{d}^{c m}(j), c_{n}^{c m}(j), \ell^{c m}(j)\right\},\left\{c_{1}^{*}(j), c_{d}^{*}(j), c_{n}^{*}(j), \ell^{*}(j)\right\}$

Then, from Corollary B.2 $\ell^{*}(0)>\ell^{c m}(0) \Leftrightarrow w^{*}(0)<w^{c m}(0)$ and because the utility function is strictly increasing $2 u\left(w^{*}(0)\right)<2 u\left(w^{c m}(0)\right)$. Adding in the occupational choice indifference conditions gives, for arbitrary $j$ :

$$
\begin{aligned}
u\left(w^{*}(j)-a^{*}(j)\right)+\theta(j) u\left(a^{*}(j)\right)+(1-\theta(j)) u\left(w^{*}(j)+a^{*}(j)\right) & =2 u\left(w^{*}(0)\right) \\
& <2 u\left(w^{c m}(0)\right) \\
& =2 u\left(w^{c m}(j) \frac{2-\theta(j)}{2}\right)
\end{aligned}
$$

or equivalently, using the indifference conditions:

$$
\begin{aligned}
E_{j}\left[U\left(c_{1}^{*}(j), c_{d}^{*}(j), c_{n}^{*}(j)\right)\right] & =E_{0}\left[U\left(c_{1}^{*}(0), c_{d}^{*}(0), c_{n}^{*}(0)\right)\right] \\
& <E_{j}\left[U\left(c_{1}^{c m}(j), c_{d}^{c m}(j), c_{n}^{c m}(j)\right)\right] \\
& =E_{0}\left[U\left(c_{1}^{c m}(0), c_{d}^{c m}(0), c_{n}^{c m}(0)\right)\right]
\end{aligned}
$$

This means that $\forall j, E_{j}\left[U\left(c_{1}^{c m}(j), c_{d}^{c m}(j), c_{n}^{c m}(j)\right)\right]>E_{j}\left[U\left(c_{1}^{*}(j), c_{d}^{*}(j), c_{n}^{*}(j)\right)\right]$ just as we required.

Lemma B.3. The ratio of labor in any two occupations, one riskier than the other, is greater in the complete markets allocation than the equilibrium allocation with incomplete markets in which $b=\tau=0$ :

$\forall j, k$ such that $\theta(j)<\theta(k), \frac{\ell^{c m}(k)}{\ell^{c m}(j)}>\frac{\ell^{*}(k)}{\ell^{*}(j)}$; these distributions satisfy the monotone likelihood ratio property. 
Proof. First, suppose this is not the case, then we assume

$$
\frac{\ell^{c m}(k)}{\ell^{c m}(j)} \leq \frac{\ell^{*}(k)}{\ell^{*}(j)}
$$

Which is equivalent to

$$
\frac{\ell^{c m}(k)}{\ell^{*}(k)} \leq \frac{\ell^{c m}(j)}{\ell^{*}(j)}
$$

and by the same reasoning as in Lemma B.1 (along with parameter restriction $\gamma<1)$

$$
\frac{w^{*}(k)}{w^{c m}(k)} \leq \frac{w^{*}(j)}{w^{c m}(j)}
$$

And because we have established that $\frac{w^{c m}(k)}{w^{c m}(j)}=\frac{2-\theta(j)}{2-\theta(k)}$ that gives us that that

$$
w^{*}(k)(2-\theta(k))<w^{*}(j)(2-\theta(j))
$$

This is to say, the expected income in occupation $j$ is higher than that in $k$ and $\theta(k)$ also has more risk. The lottery associated with $\theta(j), w(j)$ dominates that of lottery $\theta(k), w(k)$. Clearly, this cannot be consistent with household's indifference.

To put this more formally, consider the sequence of inequalities:

$$
\begin{aligned}
u\left(w^{*}(j)-a^{*}(j)\right) & +\theta(j) u\left(a^{*}(j)\right)+(1-\theta(j)) u\left(w^{*}(j)+a^{*}(j)\right) \\
> & u\left(w^{*}(j)-a^{*}(k)\right)+\theta(j) u\left(a^{*}(k)\right)+(1-\theta(j)) u\left(w^{*}(j)+a^{*}(k)\right) \\
\geq & u\left(w^{*}(k)-a^{*}(k)\right)+\theta(k) u\left(a^{*}(k)\right)+(1-\theta(k)) u\left(w^{*}(k)+a^{*}(k)\right)
\end{aligned}
$$

This is a contradiction of the household-indifference condition across occupations. The first holds by definition of $a^{*}(j), a^{*}(k)$ via the Maximum Theorem and the second holds because of our contradictory assumption. However, by our occupational-choice indifference condition, B.20,

$$
\begin{aligned}
u\left(w^{*}(j)-a^{*}(j)\right)+\theta(j) & u\left(a^{*}(j)\right)+(1-\theta(j)) u\left(w^{*}(j)+a^{*}(j)\right) \\
& =u\left(w^{*}(k)-a^{*}(k)\right)+\theta(k) u\left(a^{*}(k)\right)+(1-\theta(k)) u\left(w^{*}(k)+a^{*}(k)\right)
\end{aligned}
$$


Therefore, $\ell *$ and $\ell^{c m}$ have the monotone likelihood ratio property, with $\ell^{c m}$ dominating $\ell^{*}$

3.4 ]Proof of 3.4 . (reprinted here): Let $\ell^{*}(j)$ satisfy Definition 3.1 for the case $b=\tau=0$. Let $\ell^{c m}(j)$ be the feasible, output-maximizing allocation. Then,

$$
\int_{j=0}^{t} \ell^{*}(j) d j \leq \int_{j=0}^{t} \ell^{c m}(j) d j \quad \forall t \in(0, J]
$$

The efficient distribution of labor across occupations first-order stochastically dominates the distribution in the competitive allocation.

Proof. This is an application of the monotone likelihood ratio property shown in Lemma B.3. Given that the distributions have the monotone likelihood ratio property and $\ell^{*}(j)$ is dominated by $\ell^{c m}(j)$, this implies that $\int_{j=0}^{t} \ell^{*}(j) d j \leq$ $\int_{j=0}^{t} \ell^{c m}(j) d j \quad \forall t \in(0, J]$

B.5. Proof of existence of a strictly positive welfare maximizing level of insurance that is a Pareto improvement over no insurance.

Proof. Proof of 3.5 (reprinted here): [There exists an welfare maximizing level of insurance that is a Pareto improvement over no insurance.] Let $E U(j, \tau)$ be the expected utility $E_{j}\left[U\left(c_{1}^{*}(j), c_{n}^{*}(j), c_{d}^{*}(j)\right)\right](\tau)$ from the allocation in competitive equilibrium $\left\{c_{1}^{*}(j), c_{n}^{*}(j), c_{d}^{*}(j), a^{*}(j), n^{*}(j), \ell^{*}(j)\right\}$ satisfying Definition 3.1 for occupation-independent benefit rate $b$ funded by occupation-independent tax rate $\tau$. Then, $\exists \tau^{*}>0$ such that

$$
\frac{\partial \int \ell(j) E U\left(j, \tau^{*}\right) d j}{\partial \tau}=0
$$

and

$$
E_{j}\left[U\left(c_{1}^{*}(j), c_{n}^{*}(j), c_{d}^{*}(j)\right)\right]\left(\tau^{*}\right)>E_{j}\left[U\left(c_{1}^{*}(j), c_{n}^{*}(j), c_{d}^{*}(j)\right)\right](0) \quad \forall j
$$


We will show that $\exists j, \delta$ such that $\forall \epsilon \in(0, \delta) E U(j, \epsilon)-E U(j, 0)>0$ : It must be the case that $\exists j$ such that total earnings are higher than with zero taxes. Then, if at least one agent has higher earnings, that agent is better off and because of indifference across occupations, so too is everyone else.

We have already shown in Lemma B.7 that $\exists \delta$ such that $\forall \epsilon \in(0, \delta)$, output increases, $y(\epsilon)>y(0)$. For any $\epsilon$ within this ball, we can re-write this in terms of income:

$\int_{j=0}^{J} \ell^{*}(j, \epsilon) w(j, \epsilon)((2-\theta(j))(1-\epsilon)+\theta(j) b(\epsilon)) d j>\int_{j=0}^{J} \ell^{*}(j, 0) w(j, 0)((2-\theta(j))) d j$

This inequality might exist for one of two reasons: (1) $w(j, \epsilon)((2-\theta(j))+\theta(j) b(\epsilon))>$ $w(j, 0)((2-\theta(j)))$ for at least some $j$, or $(2) \ell^{*}(j, \epsilon)$ and $\ell^{*}(j, 0)$ are different but $\nexists j$ such that expected income is higher.

Suppose (2) holds, that is:

$$
w(j, \epsilon)((2-\theta(j))(1-\epsilon)+\theta(j) b(\epsilon)) \leq w(j, 0)(2-\theta(j)) \forall j
$$

If this is the case then we can multiply both sides of this inequality by $\ell^{*}(j, \epsilon)$

$\ell^{*}(j, \epsilon) w(j, \epsilon)((2-\theta(j))(1-\epsilon)+\theta(j) b(\epsilon)) \leq \ell^{*}(j, \epsilon) w(j, 0)(2-\theta(j))=w(j, 0) n^{*}(j, \epsilon)$

Because this holds for every $j$, we will integrate over $j$ and the inequality becomes

$$
\int \ell^{*}(j, \epsilon) w(j, \epsilon)((2-\theta(j))(1-\epsilon)+\theta(j) b(\epsilon)) d j \leq \ell^{*}(j, \epsilon) w(j, 0)(2-\theta(j))=\int w(j, 0) n^{*}(j, \epsilon) d j
$$

The left-hand side is exactly output with $\tau=\epsilon$ and we can replace $w(j, 0)$ with the solution to the firms' problem $w(j, 0)=y(0)^{1-\gamma} n^{*}(j, 0)^{\gamma-1}$.

$$
y(\epsilon) \leq y(0)^{1-\gamma} \int_{j} n^{*}(j, \epsilon) n^{*}(j, 0)^{\gamma-1} d j
$$


We can manipulate the right-hand side:

$$
\begin{aligned}
y(\epsilon) & \leq y(0)^{1-\gamma} \int_{j} n^{*}(j, \epsilon) n^{*}(j, 0)^{\gamma-1} d j \\
& =y(0)^{1-\gamma} \int_{j} \frac{n^{*}(j, \epsilon)}{n^{*}(j, 0)} n^{*}(j, 0)^{\gamma} d j \\
& =y(0)^{1-\gamma} \int_{j} \frac{n^{*}(j, \epsilon) / n^{*}(0,0)}{n^{*}(j, 0) / n^{*}(0,0)} n^{*}(j, 0)^{\gamma} d j \\
& \leq y(0)^{1-\gamma} \int_{j} \frac{n^{*}(j, \epsilon) / n^{*}(j, 0)}{n^{*}(j, 0) / n^{*}(0,0)} n^{*}(j, 0)^{\gamma} d j \\
& \leq y(0)^{1-\gamma} \int_{j} n^{*}(j, 0)^{\gamma} d j \\
& =y(0)^{1-\gamma} y(0)^{\gamma}=y(0)
\end{aligned}
$$

Where the second and third to last come from $\frac{n^{*}(j, \epsilon) / n^{*}(0,0)}{n^{*}(j, 0) / n^{*}(0,0)} \leq \frac{n^{*}(j, \epsilon) / n^{*}(0, \epsilon)}{n^{*}(j, 0) / n^{*}(0,0)} \leq$ 1, which we showed in Lemma B.5.

The result $y(\epsilon) \leq y(0)$, contradicts Lemma B.7. Thus, it must be the case that $\exists j$ such that $w(j, \epsilon)((2-\theta(j))+\theta(j) b(\epsilon))>w(j, 0)((2-\theta(j)))$

In case (1), because we have imperfect credit markets (only a storage technology, no borrowing or private insurance), it is not immediate that higher expected lifetime consumption makes workers better off. Consider the problem of a worker in occupation $j$ with some tax rate $\epsilon \in(0, \delta)$ for which $w(j, \epsilon)[(2-\theta(j))(1-\epsilon)+\theta(j) b(\epsilon)]>w(j, 0)(2-\theta(j))$. If $j=0\left(\theta_{0}=0\right)$ it is obvious that $E U_{0}(\epsilon)>E U_{0}(0)$. For all other $j$, we have

$$
w(j, 1-\epsilon)>w(j, 0)-\frac{\theta(j)}{2-\theta(j)} b(\epsilon) w(j, \epsilon)
$$

This implies for $\epsilon$ sufficiently small:

$$
\begin{aligned}
E U_{j}(\epsilon)> & \max _{a} u\left(w(j, 0)-\frac{\theta(j)}{2-\theta(j)} b(\epsilon) w(j, \epsilon)-a\right) \\
& +\theta(j) u(b(\epsilon) w(j, \epsilon)+a)+(1-\theta(j)) u\left(w(j, 0)-\frac{\theta(j)}{2-\theta(j)} b(\epsilon) w(j, \epsilon)+a\right)
\end{aligned}
$$

Then, for the marginal introduction of $b(\tau) w(j, \tau)$ we have can differentiate with respect to the benefit $b(\epsilon) w(j, \epsilon)$ at $\epsilon=0$ 


$$
\begin{aligned}
\frac{d E U_{j}(0)}{d(b w(j, 0))} & >-\frac{\theta(j)}{2-\theta(j)} u^{\prime}\left(w(j, 0)-a^{*}(j, 0)\right)+\theta(j) u^{\prime}\left(a^{*}(j, 0)\right)-\frac{\theta(j)}{2-\theta(j)}(1-\theta(j)) u^{\prime}\left(w(j, 0)+a^{*}\right. \\
& =\theta(j) u^{\prime}\left(a^{*}(j, 0)\right)-\frac{\theta(j)}{2-\theta(j)}\left[u^{\prime}\left(w(j, 0)-a^{*}(j, 0)\right)+(1-\theta(j)) u\left(w(j, 0)+a^{*}(j, 0)\right)\right] \\
& >\theta(j)\left[u^{\prime}\left(a^{*}(j, 0)\right)-u^{\prime}\left(w(j, 0)-a^{*}(j, 0)\right)\right] \\
& >0
\end{aligned}
$$

where $a^{*}(j, 0)$ is the optimizing policy at $b w(j, \tau)=0$. The second inequality comes from $u(\cdot)$ being concave. The final inequality comes from $a^{*}(j, 0)<w(j, 0)-a^{*}(j, 0)$ since $\theta(j) \in\left[0, \frac{1}{2}\right]$, which implies $u^{\prime}\left(a^{*}(j, 0)\right)>$ $u^{\prime}\left(w(j, 0)-a^{*}(j, 0)\right)$.

B.6. Supporting lemmas for $\tau>0$ is welfare improving

Lemma B.4. For wages $w(j)$, benefits $b(\tau)$ and tax rate $\tau$ and some occupational choices $\ell(j)$ and wages $w(j)$, the benefit is greater than the tax rate: $b(\tau)>\tau$.

Proof. Given the tax rate $\tau$, the government budget constraint holds that

$$
b(\tau) \int_{j=0}^{J} \theta(j) w(j) \ell(j) d j=\tau \int_{j=0}^{J}(2-\theta(j)) w(j) \ell(j) d j
$$

Then for $\theta(j)<2-\theta(j)$, the whole valid domain of $\theta, b>\tau$

Lemma B.5. Given the competitive equilibrium allocation $\left\{c_{1}^{*}(j, \tau), c_{n}^{*}(j, \tau), c_{d}^{*}(j, \tau), a^{*}(j, \tau), n^{*}(j, \tau), \ell^{*}(j, \tau)\right\}$ with tax rate $\tau$,

$$
\left.\frac{\partial}{\partial \tau}\left(\frac{\ell^{*}(j, \tau)}{\ell^{*}(0, \tau)}\right)\right|_{\tau=0}>0
$$

Proof. To sign the derivative, we will show that $\exists \delta$ such that $\forall \epsilon \in(0, \delta)$, $\left(\frac{\ell^{*}(j, \epsilon)}{\ell^{*}(0, \epsilon)}\right)>\left(\frac{\ell^{*}(j, 0)}{\ell^{*}(0,0)}\right)$. This $\delta$ can be anything small enough such that $a^{*}(j, \delta)>$ 0 for every $j$. That is to say, the borrowing constraint does not bind and choices are all in the interior of the budget set. 
Holding wage constant, for $j>0$ the expected income at $\tau=0$ and $\epsilon>0$ is $(2-\theta(j)) w(j, 0)$ and $(2-\theta(j)) w(j, 0)(1-\epsilon)+\theta(j) \hat{b}(\epsilon \mid 0) w(j, 0)$. We define $b(\epsilon)$ as the level of benefits implied by $\tau=\epsilon$ and $w(j, \epsilon)$.

The expected income $(2-\theta(j)) w(j, 0)<(2-\theta(j)) w(j, 0)(1-\epsilon)+\theta(j) \hat{b}(\epsilon) w(j, 0)$ because of Lemma B.4 and therefore, by the Maximum Theorem, expected utility is higher:

$$
\begin{gathered}
\max _{a_{\epsilon}} u\left(w(j, 0)(1-\epsilon)-a_{\epsilon}\right)+\theta(j) u\left(w(j, 0) b(\epsilon)+a_{\epsilon}\right)+(1-\theta(j)) u\left(w(j, 0)(1-\epsilon)+a_{\epsilon}\right) \\
>\max _{a_{0}} u\left(w(j, 0)-a_{0}\right)+\theta(j) u\left(a_{0}\right)+(1-\theta(j)) u\left(w(j, 0)+a_{0}\right)
\end{gathered}
$$

For $j=0$, however, the expected utility is lower with the $\epsilon$ tax rate:

$$
2 u(w(0,0)(1-\epsilon))<2 u(w(0,0))
$$

Based on indifference under $\tau=0$, we have

$$
\begin{aligned}
\max _{a_{\epsilon}} & u\left(w(j, 0)(1-\epsilon)-a_{\epsilon}\right)+\theta(j) u\left(w(j, 0) b(\epsilon)+a_{\epsilon}\right)+(1-\theta(j)) u\left(w(j, 0)(1-\epsilon)+a_{\epsilon}\right) \\
> & 2 u(w(0,0)(1-\epsilon))
\end{aligned}
$$

But indifference with $\tau=\epsilon$ implies that $E U(j, \epsilon)=E U(0, \epsilon)$. Therefore, $w(j, \epsilon) \neq w(j, 0)$ and $\frac{w(j, \epsilon)}{w(0, \epsilon)} \neq \frac{w(j, 0)}{w(0,0)}$

We now suppose the contradiction to our premise, that $\exists \hat{j}$ such that

$$
\frac{\ell^{*}(\hat{j}, \epsilon)}{\ell^{*}(0, \epsilon)} \leq \frac{\ell^{*}(\hat{j}, 0)}{\ell^{*}(0,0)} \Leftrightarrow \frac{w(\hat{j}, \epsilon)}{w(0, \epsilon)} \geq \frac{w(\hat{j}, 0)}{w(0,0)}
$$

As we have just shown in Equation B.33, it cannot be that $\frac{w(\hat{j}, \epsilon)}{w(0, \epsilon)}=\frac{w(\hat{j}, 0)}{w(0,0)}$, therefore

$$
\frac{w(\hat{j}, \epsilon)}{w(0, \epsilon)}>\frac{w(\hat{j}, 0)}{w(0,0)}
$$

But then, $\exists k$ such that $k>j$ but

$$
\frac{w(\hat{j}, \epsilon)}{w(0, \epsilon)}>\frac{w(k, \epsilon)}{w(0, \epsilon)}>\frac{w(\hat{j}, 0)}{w(0,0)}
$$


which means that $w(\hat{j}, \epsilon)>w(k, \epsilon)$ and therefore $(2-\theta(\hat{j})) w(\hat{j}, \epsilon)(1-\epsilon)+\theta(\hat{j}) b(\epsilon) w(\hat{j}, \epsilon)>(2-\theta(k)) w(k, \epsilon)(1-\epsilon)+\theta(k) b(\epsilon) w(k, \epsilon)$

But if $k$ is a riskier occupation with lower expected income than $\hat{j}$, then workers cannot be indifferent. For any such $\hat{j}$ we could find a $k$ and therefore $\forall j$ :

$$
\frac{w(j, \epsilon)}{w(0, \epsilon)}<\frac{w(j, 0)}{w(0,0)} \Leftrightarrow \frac{\ell^{*}(j, \epsilon)}{\ell^{*}(0, \epsilon)}<\frac{\ell^{*}(j, 0)}{\ell^{*}(0,0)}
$$

Corollary B.6. The mass in the risk-free occupation, where $\theta(0)=0$, is decreasing in $\tau:\left.\frac{\partial \ell^{*}(0, \tau)}{\partial \tau}\right|_{\tau=0}<0$

Proof. This is a direct result of Lemma B.5 and the identity $\int_{j} \ell^{*}(j, \tau) d j=1$. If $\exists \delta: \forall \epsilon \in(0, \delta) \frac{\ell^{*}(j, \epsilon)}{\ell^{*}(0, \epsilon)}>\frac{\ell^{*}(j, 0)}{\ell^{*}(0,0)}$. Consider integrating over $j$, then

$$
\begin{aligned}
\int_{j} \frac{\ell^{*}(j, \epsilon)}{\ell^{*}(0, \epsilon)} d j & >\int_{j} \frac{\ell^{*}(j, 0)}{\ell^{*}(0,0)} d j \\
\frac{\int_{j} \ell^{*}(j, \epsilon) d j}{\int_{j} \ell^{*}(j, 0) d j} & >\frac{\ell^{*}(0, \epsilon)}{\ell^{*}(0,0)} \\
1 & >\frac{\ell^{*}(0, \epsilon)}{\ell^{*}(0,0)}
\end{aligned}
$$

Lemma B.7. Let, $y^{*}(\tau)$ be the output at competitive equilibrium allocation $\left\{c_{1}^{*}(j, \tau), c_{n}^{*}(j, \tau), c_{d}^{*}(j, \tau), a^{*}(j, \tau) n^{*}(j, \tau), \ell^{*}(j, \tau)\right\}$ with tax rate $\tau . y^{*}(\tau)$ is increasing in $\tau$ at $\tau=0,\left.\frac{\partial y}{\partial \tau}\right|_{\tau=0}>0$

Proof. Recall the tax-free competitive equilibrium has lower output than the first best planner's efficient output, $y^{*}(0) \leq y^{c m}$ (as shown in 3.4) and that this is because the distribution of labor in the first best first order stochastically dominate the distribution of labor in the tax-free competitive equilibrium: $\int_{j=0}^{x} n^{*}(j, 0) d j \geq \int_{j=0}^{x} n^{c m}(j, 0) d j$ for all $x \in(0, J]$. Here, we use the 
related Lemma that helped establish monotone likelihood ratio dominance, B.3 and the similar Lemma relating $\tau>0$ to $\tau=0$, Lemma B.5. Using these, for any $j>0$, we can find an $v(j)>0$ small enough such that:

$$
\frac{\ell^{*}(j, 0)}{\ell^{*}(0,0)}<\frac{\ell^{*}(j, v(j))}{\ell^{*}(0, v(j))}<\frac{\ell^{c m}(j)}{\ell^{c m}(0)}
$$

Let $\delta=\min _{j} v(j)$ so that $\forall \epsilon \in(0, \delta)$ and $\forall j$,

$$
\frac{\ell^{*}(j, 0)}{\ell^{*}(0,0)}<\frac{\ell^{*}(j, \epsilon)}{\ell^{*}(0, \epsilon)}<\frac{\ell^{c m}(j)}{\ell^{c m}(0)}
$$

We now treat cases of $\gamma>0$ and $\gamma<0$ separately, though the steps are exactly the same. First, with $\gamma>0$, we multiply Inequality B.34 by $(2-\theta(j))$ and exponentiate to $\gamma$.

$$
\left(\frac{(2-\theta(j)) \ell^{*}(j, 0)}{\ell^{*}(0,0)}\right)^{\gamma}<\left(\frac{(2-\theta(j)) \ell^{*}(j, \epsilon)}{\ell^{*}(0, \epsilon)}\right)^{\gamma}<\left(\frac{(2-\theta(j)) \ell^{c m}(j)}{\ell^{c m}(0)}\right)^{\gamma}
$$

Because this holds for all $j$, we integrate over $j$ and then exponentiate to $\frac{1}{\gamma}$

$$
\begin{aligned}
& \left(\int_{j}\left(\frac{(2-\theta(j)) \ell^{*}(j, 0)}{\ell^{*}(0,0)}\right)^{\gamma} d j\right)^{\frac{1}{\gamma}}<\left(\int_{j}\left(\frac{(2-\theta(j)) \ell^{*}(j, \epsilon)}{\ell^{*}(0, \epsilon)}\right)^{\gamma} d j\right)^{\frac{1}{\gamma}}<\left(\int_{j}\left(\frac{(2-\theta(j)) \ell^{c m}(j)}{\ell^{c m}(0)}\right)^{\gamma} d j\right) \\
& \frac{y^{*}(0)}{\ell^{*}(0,0)}<\frac{y^{*}(\epsilon)}{\ell^{*}(0, \epsilon)}<\frac{y^{c m}}{\ell^{c m}(0)}
\end{aligned}
$$

If $\gamma<0$ we have the same, but inequalities flip twice when we exponentiate:

$$
\begin{aligned}
& \frac{\ell^{*}(j, 0)}{\ell^{*}(0,0)}<\frac{\ell^{*}(j, \epsilon)}{\ell^{*}(0, \epsilon)}<\frac{\ell^{c m}(j)}{\ell^{c m}(0)} \\
& \left(\frac{(2-\theta(j)) \ell^{*}(j, 0)}{\ell^{*}(0,0)}\right)^{\gamma}>\left(\frac{(2-\theta(j)) \ell^{*}(j, \epsilon)}{\ell^{*}(0, \epsilon)}\right)^{\gamma}>\left(\frac{(2-\theta(j)) \ell^{c m}(j)}{\ell^{c m}(0)}\right)^{\gamma} \\
& \left(\int_{j}\left(\frac{(2-\theta(j)) \ell^{*}(j, 0)}{\ell^{*}(0,0)}\right)^{\gamma} d j\right)^{\frac{1}{\gamma}}<\left(\int_{j}\left(\frac{(2-\theta(j)) \ell^{*}(j, \epsilon)}{\ell^{*}(0, \epsilon)}\right)^{\gamma} d j\right)^{\frac{1}{\gamma}}<\left(\int_{j}\left(\frac{(2-\theta(j)) \ell^{c m}(j)}{\ell^{c m}(0)}\right)^{\gamma} d j\right) \\
& \frac{y^{*}(0)}{\ell^{*}(0,0)}<\frac{y^{*}(\epsilon)}{\ell^{*}(0, \epsilon)}<\frac{y^{c m}}{\ell^{c m}(0)}
\end{aligned}
$$


Now we have to use Lemma B.1 and Corollary B.6 to order $\ell^{*}(0,0), \ell^{*}(0, \epsilon)$ and $\ell^{c m}(0)$. This tells us $\ell^{*}(0,0)>\ell^{*}(0, \epsilon)>\ell^{c m}(0)$ and therefore

$$
\frac{1}{\ell^{*}(0,0)}<\frac{1}{\ell^{*}(0, \epsilon)}<\frac{1}{\ell^{c m}(0)}
$$

Therefore, we can re-write $\frac{y^{*}(0)}{\ell^{*}(0,0)}<\frac{y^{*}(\epsilon)}{\ell^{*}(0, \epsilon)}$ as $\ell^{*}(0,0) y^{*}(0)<y^{*}(\epsilon) \frac{\ell^{*}(0,0)}{\ell^{*}(0, \epsilon)}$ and because $y^{*}(\epsilon) \frac{\ell^{*}(0,0)}{<} 1$ this implies

$$
y^{*}(0)<y *(\epsilon) .
$$

\section{B.7. Achieving the first best allocation}

Proof. Proof of 3.6 (reprinted here): [The welfare maximizing level of social insurance with occupation independent tax and benefit rates does not maximize output.] Let $n^{c m}(j)$ characterize the efficient (output maximizing) allocation. Let $\left\{n^{r p}(j), w^{r p}(j)\right\} ; \tau, b$ be the constrained optimal planner allocation (maximizes welfare in competitive equilibrium given policy tools). Then $y^{r p}<y^{c m}$.

We will show the competitive equilibrium indifference condition $E_{j}[U(w(j))]=$ $E_{0}[U(w(0))]$ is not satisfied at the output maximizing allocation. Recall, wages at the output maximizing allocation satisfy:

$$
w(j)=\frac{2}{2-\theta(j)} w(0) \quad \forall j
$$

Therefore, we can write $E_{j}[U(w(j))]$ for arbitrary $j \in(0, J]$ as:

$$
\begin{aligned}
E_{j}[U(w(j))] & =\max _{a}\left\{u\left((1-\tau-a) \frac{2}{2-\theta(j)} w_{0}\right)+\right. \\
& +\theta(j) u\left((b+a) \frac{2}{2-\theta(j)} w_{0}\right)+(1-\theta(j)) u\left((1-\tau+a) \frac{2}{2-\theta(j)} w_{0}\right)
\end{aligned}
$$


By concavity of $u(\cdot)$, it must be $c_{1} \leq E\left[c_{2}\right]$. Some algebra delivers:

$$
\begin{aligned}
c_{1} \leq E\left[c_{2}\right] & \Rightarrow(1-\tau-a) \leq a+\theta(j) b+(1-\theta(j))(1-\tau) \\
& \Rightarrow a \geq \frac{\theta(j)}{2}(1-\tau-b) \\
& \Rightarrow \quad c_{1} \leq w(0) \frac{2}{2-\theta(j)} \frac{(2-\theta(j))(1-\tau)+\theta(j) b}{2} \\
& \Rightarrow c_{1} \leq w(0)\left[(1-\tau)-\frac{\theta_{j}}{2-\theta_{j}} b\right]
\end{aligned}
$$

This inequality implies first period consumption in occupation $j$ is less than that of the risk free occupation: $c_{1}^{*}(j) \leq(1-\tau) w(0)$; if:

$$
\left[(1-\tau)-\frac{\theta_{j}}{2-\theta_{j}} b\right] \leq(1-\tau)
$$

This is clearly the case since $\theta \in(0, J]$ and $b \geq 0$. Thus, we have shown $c_{1}(j)<c_{1}(0)$. Jensen's inequality and the Euler, $u^{\prime}\left(c_{1}\right)=E\left[u^{\prime}\left(c_{2}\right)\right]$ imply that $u\left(c_{1}\right)>E_{j}\left[u\left(c_{2}\right)\right]$ and together we have:

$$
E_{0}[u(w(0))]=2 u(w(0)(1-\tau)) \geq 2 u\left(c_{1}(j)\right)>E_{j}[u(w(j))]
$$

This contradicts the occupation indifference condition and so productive efficiency cannot be maintained as a competitive equilibrium when using a proportional tax and benefit scheme with occupation independent rates.

Proof. Proof of 3.7 (reprinted here): [The first-best planner allocation can be achieved with a lump-sum or proportional tax and occupation-specific benefits.] Let $\mathcal{A}^{*}(\widehat{b}, \widehat{\tau})=\left\{c_{1}^{*}(j), c_{n}^{*}(j), c_{d}^{*}(j), a^{*}(j), n^{*}(j), \ell^{*}(j)\right\}$ satisfying Definition 3.1 given arbitrary occupation-specific benefits $b(j)$ funded by occupation-specific taxes $\tau(j)$. Let $\mathcal{A}^{c m}=\left\{c_{1}^{c m}(j), c_{n}^{c m}(j), c_{d}^{c m}(j), a^{c m}(j), n^{c m}(j) \ell^{c m}(j)\right\}$ define the first-best planner's allocation. Then, $\exists b(j), \tau(j)$ such that $\mathcal{A}^{*}(b((j)), \tau(j))=$ $\mathcal{A}^{c m}$

To achieve the efficient allocation in the decentralized economy, $w(j)=$ $w\left(j^{\prime}\right)$ for all $j, j^{\prime}$ and $E U_{j}=E U_{j^{\prime}}$. This can be achieved with occupation 
specific benefits and a non-occupation specific tax. These can be any combination of lump-sum or proportional. Here consider lump-sum benefit and proportional tax. The constrained optimal planner chooses:

$$
\begin{aligned}
b(j) & =w(j)(1-\tau) \\
\tau & =\frac{\int_{j} n^{c m}(j) \theta(j) b(j) d j}{Y}
\end{aligned}
$$

Here, individuals in each occupation consume $w(j)(1-\tau)$ in each period, regardless of disability status. Then for $E U_{j}=E U_{j^{\prime}}$, it must be $w(j)=$ $w\left(j^{\prime}\right)$ for all $j, j^{\prime}$ and thus the constrained optimal planner achieves welfare maximization and output maximization simultaneously.

\section{B.8. Extension: Heterogeneous Beta and Occupational Sorting}

Here we expand the model to exhibit the kind of sorting on individual heterogeneity which we worked to control for in the empirical section. Specifically, we consider heterogeneity in the discount factor. We provide proofs for a population with two-types: patient types with high-beta $\beta^{H}$ and impatient types with low-beta $\beta^{L}$. The measure of each type is fixed in each generation: $\phi^{L} \geq 0, \phi^{H} \geq 0$ such that $\phi^{L}+\phi^{H}=1 .{ }^{13}$

Our goal is to show the following. First, more patient individuals sort into higher risk occupations. Second, the main results of the paper go through: (i) the laissez-faire allocation is inefficient; and (ii) an economy with the marginal introduction of SDI from zero generates higher welfare for all agents compared to an economy with no SDI. We discuss interesting implications for unequal gains from SDI based on these results in the text.

The Social Planner's Problem.. The allocation of labor in the social planner's problem is unchanged. The ratio of labor in two occupations are proportional to their risks.

\footnotetext{
${ }^{13}$ Our results do generalize to $N$ types.
} 
Step 1: Separation of low and high-beta occupations. Want to show that the more patient high-beta types sort into the least risky occupations. To do this, we will show that if the high-beta type is indifferent between the risk-free occupation and any risky occupation, then the low-beta type strictly prefers the risky occupation. Thus, the low-beta type must inhabit strictly riskier occupations.

Proposition B.8 (Monotone Occupational Sorting). There exists a unique $\bar{j}$ such that any occupation with $j<\bar{j}$ employs only high-beta types and any occupation with $j>\bar{j}$ employs only low-beta types.

Proof. Observe that for arbitrary occupations $j<k$ that employ a highbeta type, it must be: $E_{j}\left[U^{h}\right]=E_{k}\left[U^{h}\right]$; and the same condition holds for low-beta types. Now, want to show for arbitrary occupations $j<k$ such that $E_{j}\left[U^{h}\right]=E_{k}\left[U^{h}\right]$, it must be $E\left[U^{\ell}(j)\right]<E\left[U^{\ell}(k)\right]$, which implies that occupation $j$ will not employ a low-beta type.

WLOG assume $\beta^{H}=1$ and denote $\beta^{L}$ as just $\beta$. Let high-types be indifferent between occupations $j<k$.

$$
\begin{aligned}
u\left(w^{*}(k)-a^{* H}(k)\right)+\theta(k) u\left(a^{* H}(k)\right)+(1-\theta(k)) u\left(w^{*}(k)+a^{* H}(k)\right) & =u\left(w^{*}(j)-a^{* H}(j)\right)+\theta(j) u\left(a^{* H}(j\right. \\
& =2 u\left(w^{*}(0)\right)
\end{aligned}
$$

Want to show:

$u\left(w^{*}(j)-a^{* L}(j)\right)+\beta \theta(j) u\left(a^{* L}(j)\right)+\beta(1-\theta(j)) u\left(w^{*}(k)+a^{* L}(j)\right)>(1+\beta) u\left(w^{*}(0)\right)$

Add $(1-\beta) u\left(w^{*}(0)\right)$ to both sides. Suffices to verify:

$$
\begin{aligned}
& u\left(w^{*}(j)-a^{* L}(j)\right)+\beta \theta(j) u\left(a^{* L}(j)\right)+\beta(1-\theta(j)) u\left(w^{*}(j)+a^{* L}(j)\right)+(1-\beta) u\left(w^{*}(0)\right) \\
> & (1+\beta) u\left(w^{*}(0)\right)+(1-\beta) u\left(w^{*}(0)\right) \\
= & u\left(w^{*}(j)-a^{* H}(j)\right)+\theta(j) u\left(a^{* H}(j)\right)+(1-\theta(j)) u\left(w^{*}(j)+a^{* H}(j)\right)
\end{aligned}
$$


By optimality of $a^{* L}(j)$, we have:

$$
\begin{aligned}
& \left.u\left(w^{*}(j)-a^{* L}(j)\right)+\beta \theta(j) u\left(a^{* L}(j)\right)+\beta(1-\theta(j)) u\left(w^{*}(j)+a^{* L}(j)\right)+(1-\beta) u\left(w^{*}(0)\right)\right) \\
\geq & u\left(w^{*}(j)-a^{* H}(j)\right)+\beta \theta(j) u\left(a^{* H}(j)\right)+\beta(1-\theta(j)) u\left(w^{*}(j)+a^{* H}(j)\right)+(1-\beta) u\left(w^{*}(0)\right)
\end{aligned}
$$

It then suffices to show

$$
\begin{aligned}
& u\left(w^{*}(j)-a^{* H}(j)\right)+\beta \theta(j) u\left(a^{* H}(j)\right)+\beta(1-\theta(j)) u\left(w^{*}(j)+a^{* L}(j)\right)+(1-\beta) u\left(w^{*}(0)\right) \\
> & u\left(w^{*}(j)-a^{* H}(j)\right)+\theta(j) u\left(a^{* H}(j)\right)+(1-\theta(j)) u\left(w^{*}(j)+a^{* H}(j)\right)
\end{aligned}
$$

Algebraically, this inequality holds if and only if $u\left(w^{*}(0)\right)>\theta(j) u\left(a^{* H}(j)\right)+$ $(1-\theta(j)) u\left(w^{*}(j)+a^{* H}(j)\right)$ But, by assumption,

$2 u\left(w(0)^{*}\right)=u\left(w^{*}(j)-a^{* H}(j)\right)+\theta(j) u\left(a^{* H}(j)\right)+(1-\theta(j)) u\left(w^{*}(j)+a^{* H}(j)\right)$

We have our result if $u\left(w^{*}(j)-a^{* H}(j)\right)>\theta(j) u\left(a^{* H}(j)\right)+(1-\theta(j)) u\left(w^{*}(j)+\right.$ $\left.a^{* H}(j)\right)$. Suppose not, then by the concavity of $u$ it must be, $a^{* H}(j)>$ $w^{*}(j)-a^{* H}(j) \Rightarrow a^{* H}(j)>\frac{1}{2} w^{*}(j)$. But this violates the first order condition:

$$
\begin{aligned}
u^{\prime}\left(w^{*}(j)-a^{* H}(j)\right) & \geq u^{\prime}\left(\frac{1}{2} w^{*}(j)\right) \\
& \geq \theta(j) u^{\prime}\left(\frac{1}{2} w^{*}(j)\right)+(1-\theta(j)) u^{\prime}\left(3 / 2 w^{*}(j)\right)
\end{aligned}
$$

Step 2: CM with Heterogenous Beta is Pareto Inefficient. In this section we use the result from the previous proposition to argue that the competitive allocation is inefficient both in terms of consumption sharing and occupational sorting. In particular, we will show it is inefficient within beta-types, a simple extension of our proofs for the homogenous beta case.

Proposition B.9 (The Competitive Allocation with Heterogenous Beta and without Social Insurance is Pareto Inefficient). Let $\left\{c_{1}^{*}(j), c_{n}^{*}(j), c_{d}^{*}(j)\right.$, 
$\left.a^{*}(j), n^{*}(j), \ell^{*}(j)\right\}_{j=1}^{J}$ satisfy the definition of a competitive equilibrium in the case of (i) a continuum of occupations; (ii) two types of agents with different discount factors; (ii) and no social insurance $(b=\tau=0)$. There exists an alternative feasible allocation $\left\{\hat{c}_{1}(j), \hat{c}_{d}(j), \hat{c}_{n}(j), \hat{\ell}(j)\right\}_{j=1}^{J}$ that:

(i) increases expected utility in each occupation

$$
\begin{aligned}
& E_{j}\left[U^{m}\left(\hat{c}_{1}(j), \hat{c}_{n}(j), \hat{c}_{d}(j)\right)\right] \geq E_{j}\left[U^{m}\left(c_{1}^{*}(j), c_{n}^{*}(j), c_{d}^{*}(j)\right)\right] \forall j \in\{1, \ldots, J\} ; \quad m \in\{L, H\} \\
\exists k \text { s.t. } & E_{k}\left[U\left(\hat{c}_{1}(k), \hat{c}_{n}(k), \hat{c}_{d}(k)\right)\right]>E_{k}\left[U\left(c_{1}^{*}(k), c_{n}^{*}(k), c_{d}^{*}(k)\right)\right]
\end{aligned}
$$

(ii) is feasible

$$
\int_{j} \hat{\ell}(j)\left(\hat{c}_{1}(j)+\theta(j) \hat{c}_{d}(j)+(1-\theta(j)) \hat{c}_{n}(j)\right) d j \leq\left(\int_{j}(\hat{\ell}(j)(2-\theta(j)))^{\gamma} d j\right)^{\frac{1}{\gamma}}
$$

Proof. This is an application of Proposition 3.3. Fix $\bar{j}$ dictating the span of occupations of each type in the LF economy according to the above monotone sorting proposition. Next, open complete markets within each beta type and allow occupation choices within $j \in[\bar{j}, J)$ for the low-beta types and $j \in[0, \bar{j})$ for the high-beta types. By Proposition 3.3, the allocation will be preferred by each type and increase output.

Proposition B.10 (The Competitive Allocation with Heterogenous Beta and without Social Insurance is Puts too Few Workers in Risky Occupations). Let $\left\{\ell_{j}^{*}\right\}$ satisfy the definition of a competitive equilibrium in the case of (i) a continuum of occupations; (ii) two types of agents with different discount factors; (ii) and no social insurance $(b=\tau=0)$. Let $\left\{\ell_{j}^{C M}\right\}$ be the feasible output maximizing allocation. Then:

$$
\int_{j=0}^{t} \ell_{j}^{*} \leq \int_{j=0}^{t} \ell_{j}^{C M} \quad \forall t \in[0, J]
$$

ie: the efficient distribution of labor across occupations first-order stochastic dominates the distribution in the competitive equilibrium. 
Proof. Fix $\bar{j}$ dictating the span of occupations of each type in the LF economy according to the above monotone sorting proposition. It is a simple application of Proposition 3.4 to show first order stochastic dominance within the occupations held by a given beta type, when normalized by the total number of workers in occupations either greater or less than $\bar{j}$.

$$
\begin{gathered}
\frac{\int_{j=0}^{t} \ell_{j}^{*}}{\int_{j=0}^{\bar{j}} \ell_{j}^{*}} \leq \frac{\int_{j=0}^{t} \ell_{j}^{C M}}{\int_{j=0}^{\bar{j}} \ell_{j}^{C M}} \quad \forall t \in[0, \bar{j}] \\
\frac{\int_{j=\bar{j}}^{s} \ell_{j}^{*}}{\int_{j=\bar{j}}^{J} \ell_{j}^{*}} \leq \frac{\int_{j=\bar{j}}^{s} \ell_{j}^{C M}}{\int_{j=\bar{j}}^{J} \ell_{j}^{C M}} \quad \forall s \in(\bar{j}, J]
\end{gathered}
$$

What remains to show is that the LF economy puts more workers in the less risk occupations, those below $\bar{j}$, than the efficient allocation:

$$
\phi^{*} \equiv \int_{j=0}^{\bar{j}} \ell_{j}^{*} \geq \int_{j=0}^{\bar{j}} \ell_{j}^{c m}
$$

where $\phi^{*}$ equals the measure of high-beta type agents in each generation. Given B.35, it is sufficient to show $\ell^{*}(0)>\ell^{c m}(0)$ to show B.37 holds. This follows the same direct argument as the baseline case since, again, we are only dealing with relative quantities within a $\beta$ type.

Step 3: Welfare Gain from SDI w/ Heterogenous Beta. The prior two steps have shown the competitive allocation of the economy with heterogenous beta generates the same sources of inefficiency, lack of risk sharing and productive inefficiency, as the economy with just one beta type. In this final step we show the marginal introduction of social disability insurance (SDI) continues to increase welfare and does so for both beta types.

Proposition B.11 (Social Insurance is Welfare Improving (on the margin) for all Beta-Types). Let $E U^{i}(\tau)$ be the expected utility of a type $\beta^{i} \in\left\{\beta^{h}, \beta^{\ell}\right\}$ agent from the competitive equilibrium in the case of (i) a continuum of occupations; (ii) two types of agents with different discount factors; (ii) and 
proportional social insurance $\left(\tau \geq 0, b_{j}=b w_{j}\right.$, and $\sum_{j}\left(2-\theta_{j}\right) \ell_{j} \tau w_{j}=$ $\left.\sum_{j} \theta_{j} \ell_{j} b w_{j}\right)$. Then:

$$
E U^{\prime i}(0)>0 \quad \forall i \in\{\ell, h\}
$$

Proof. Lemma 1. SDI increases output $\left(\left.\frac{d y^{*}}{d \tau}\right|_{\tau=0}>0\right)$. Fix $\bar{j} \in(0, J]$. Impose a modified competitive economy with the additional restriction that high-beta types may only choose occupations $j \leq \bar{j}$ and low-beta types may only choose occupations $j>\bar{j}$. By an application of Lemma C.6, we have $\left.\frac{d y^{*}}{d \tau}\right|_{\tau=0}>0$, maintaining fixed $\bar{j}$. Denote $\bar{j}(\tau)$, as the high versus low beta cut-off in the economy with SDI characterized by $\tau \in[0, \epsilon]$ and $\bar{j}^{c m}$ as the cut-off in the first-best planner (output maximizing) economy. What remains to be shown is that:

$$
\bar{j}(0) \leq \bar{j}(\tau) \leq \bar{j}^{\mathrm{cm}}
$$

Suppose this is not true, that at marginal occupation $k$ such that occupation $k$ employs high-beta types and occupation $k+\varepsilon$ employs low-beta types for all $\varepsilon>0$, we have: $E_{j}\left[U^{H}(w(j, 0))\right]=E_{k}\left[U^{H}(w(k, 0))\right]$ for all $j<k$, but $E_{j}\left[U^{H}(w(j, \tau))\right]>E_{k}\left[U^{H}(w(k, \tau))\right]$ for some $j<k$.

At $\tau=0$, competitive wages must be monotone increasing, otherwise expected income would be higher in a less risky occupation and the occupation indifference condition cannot be satisfied. This implies that $w(k+\varepsilon, 0)>$ $w(k, 0)$. Let $k-\delta<\bar{j}(\tau)$ The assumption for contradiction that $\bar{j}(0)>\bar{j}(\tau)$ combined with Lemma C.6 imply $n(k-\delta, \tau)>n(k-\delta, 0)$ for $\delta$ small, because the allocation under $\tau$ covers fewer occupations and puts more mass at riskier occupations. Subsequently: $w(k-\delta, \tau)<w(k-\delta, 0)$. Likewise, for occupations $k$, we have assumed $k>\bar{j}(\tau)$ and by the same logic applying C.6 $n(k, \tau)<n(k, 0)$ for $\delta$ small, because the allocation under $\tau$ covers more occupations and puts more mass at riskier occupations. Subsequently: $w(k, \tau)>w(k, 0)>w(k-\delta, 0)>w(k-\delta, \tau)$.

Let $E_{j}(y(w(j), \tau))$ be the total expected income in occupation $j$ under 
SDI scheme $\tau$. We then have:

$$
\begin{aligned}
E_{k}(y(w(k), \tau)) & =(2-\theta(k)) w(k, \tau)(1-\tau)+\theta(k) w(k, \tau) b(\tau)> \\
>E_{k}(y(w(k), 0)) & =(2-\theta(k)) w(k, 0)> \\
E_{k-\delta}(y(w(k-\delta), 0)) & =(2-\theta(k-\delta)) w(k-\delta, 0)
\end{aligned}
$$

Where the first inequality is true for $\tau$ small since $b(\tau)>\tau$ at all values and $w(k, \tau)>w(k, 0)>w(k-\delta, 0)$. But, we have assumed $E_{k}\left[U^{H}(w(k, 0))\right]=$ $E_{k-\delta}\left[U^{H}(w(k-\delta, 0))\right]$, then occupation $k$ at tax rate $\tau$ represents a lottery with less risk and a strictly higher risk premium relative to occupation $k-\delta$ and it must be: $E_{k}\left[U^{H}(w(k, \tau))\right]>E_{k-\delta}\left[U^{H}(w(k-\delta, \tau))\right]$, a contradiction.

Part 2. SDI increases welfare for both types. Given the prior lemmas, proofs from the baseline case can be applied directly for high-beta types. What remains is to show low-beta types also gain. The thrust is similar to the baseline: so long as $\tau$ is sufficiently small that full insurance is not provided $\left(a^{*}(j, \tau)>0\right.$ for all $\left.j\right)$ and output increases, then we have a welfare improvement at $\tau$ for all types.

First, we argue that the introduction of disability insurance raises expected consumption of the workers in the highest risk occupation, $j=J$. Expected consumption in occupation $j$ for an arbitrary $\tau$ is:

$$
\begin{aligned}
C(j) & \equiv w_{j}\left[(1-\tau)\left(2-\theta_{j}\right)+b \theta_{j}\right] \\
& =n_{j}^{\gamma-1} y^{1-\gamma}\left[(1-\tau)+\frac{\theta}{2-\theta} b\right]
\end{aligned}
$$

The second equality comes following some algebra after substitution of the competitive wage: $w_{j}=\left(\frac{y}{n_{j}}\right)^{-1 \gamma}$. The marginal change in total consumption at the introduction of SDI is:

$$
\begin{aligned}
\frac{d C(j)}{d \tau} \|_{\tau=0} & =\frac{d n_{j}}{d \tau}(1-\gamma)\left(n_{j}\right)^{\gamma-2} y^{1-\gamma}+(1-\gamma) y^{-\gamma} n_{j}^{\gamma-1} \frac{d y}{d \tau}+n_{j}^{\gamma-1} y^{1-\gamma}\left[\frac{\theta_{j}}{2-\theta_{j}} \frac{d b}{d \tau}-1\right] \\
& =\gamma w_{j} \frac{d n_{j}}{d \tau}+(1-\gamma) y^{-\gamma} n_{j}^{\gamma} \frac{d y}{d \tau}+n_{j} w_{j}\left[\frac{2}{2-\theta_{j}} \frac{d b}{d \tau}-1\right]
\end{aligned}
$$


Recall: $\quad b=\tau \frac{y}{\int_{j} \theta(j) w(j) \ell(j) d j}$. Since $\theta_{j} \in[0, J]$, the highest possible dependency ratio is one to three, and so we know $b \geq 3 \tau$. Additionally, we have the prior results that (1) $n(\tau) \succ_{f s d} n(0)$ and so $\left.\frac{d n(J)}{d \tau}\right|_{\tau=0}>0$; and (2) $\left.\frac{d y}{d \tau}\right|_{\tau=0}$. Together, these things imply that, given our parameter restriction $\gamma \in(-\infty, 1)$, we have $\frac{d C(j)}{d \tau} \|_{\tau=0}>0$.

Now we argue $\frac{d E_{J}[C(J)]}{d \tau} \|_{\tau=0}>0$ implies $\left.\frac{d E_{J} U(w(J, \tau))}{d \tau}\right|_{\tau=0}>0$ which together with (1) the occupation indifference condition combined with (2) the sorting of low-beta types into the highest risk occupations; will imply $\left.\frac{d E_{j} U(w(j, \tau))}{d \tau}\right|_{\tau=0}>0$ for all low-beta types (ie: occupations $j>\bar{j}(\tau=0)$ ).

Let the optimal choice of assets in occupation $j=J$ at no SDI $(\tau=0)$ be denoted:

$a^{*}(J, 0) \equiv \underset{a \geq 0}{\operatorname{argmax}} u((1-a) w(J, 0))+\beta \theta(J) u(a w(J, 0))+\beta(1-\theta(J)) u((1+a) w(J, 0))$

The Inada condition $\lim _{c \rightarrow 0} u^{\prime}(c)=\infty$ ensures that for all $\beta>0$, and $N \in \mathbf{N}, \exists \delta>0$ such that $\forall \quad \tau \in(0, \delta)$ we have $a^{*}(J, 0)>\frac{N}{\beta} \tau$. Then, for $N$ large, we can find a $\tau$ small such that $a^{*}(J, \tau)>0$. At such a $\tau$, Jensen's inequality provides $E_{J}[U(w(J, \tau))]>E_{J}[U(w(J, 0))]$ because the former has higher expected payoff with lower variance.

\section{B.9. Extension: Costly Disability}

Here we can further generalize the model to include a cost of becoming disabled. We will model it as a function $u^{d}(c)$ with the following properties: $u^{\prime d}(c)>u^{\prime}(c)$ and $u^{d}(c)<u(c)$ for all $\infty>c>0$. In words, this captures both an expenditure cost of disability through marginal utility and a utility cost through the absolute value. To simplify the analysis we assume further: $u^{\prime d}(c(1+\chi))=u^{\prime}(c)$ and $u^{d}(c(1+\chi))=u(c)$ for all $\infty>c>0$. That is the cost of disability is a constant proportion of consumption $(1+\chi)$ required to regain the utility of the non-disabled. In this way, we have a baseline where these costs have constant marginal relationships with the level of consumption and we can isolate factors in the planner's problem cleanly. 
Step 1: Characterization of the First Best Allocation. The problem of a firstbest social planner is similar to the base case except (i) relative consumption:

$$
\begin{aligned}
\max _{\ell(j), c_{d}(j), c_{n}(j), c_{1}(j)} & \int_{j}\left[u\left(c_{1}(j)\right)+\theta(j) u^{d}\left(c_{d}(j)\right)+(1-\theta(j)) u\left(c_{n}(j)\right)\right] d j \\
\text { st } \quad & \int_{j} \ell(j)\left[c_{1}(j)+\theta(j) c_{d}(j)+(1-\theta(j)) c_{n}(j)\right] d j \leq\left(\int_{j}(2-\theta(j))^{\gamma} \ell(j)^{\gamma} d j\right)^{1 / \gamma} \\
& \int_{j} \ell(j) d j=1
\end{aligned}
$$

We see this problem is similar to the base case except: (i) relative consumption in the disabled and non-disabled states must be chosen; and (ii) the planner considers the differential marginal utility of consumption when allocating agents.

First order conditions straight-forwardly deliver:

$$
u^{\prime}\left(c_{1}\right)=u^{\prime}\left(c_{n}\right)=u^{\prime d}\left(c_{d}\right) \quad \forall j
$$

which implies: $c \equiv c_{1}=c_{n}<c_{d}$.

Next we consider the allocation of labor across occupations. First order conditions imply:

$$
\begin{aligned}
w_{j} & =c+\frac{\theta(j)}{2-\theta(j)} c_{d} \\
\frac{w_{j}}{w_{0}} & =1+\frac{\theta(j)}{2-\theta(j)} \frac{c_{d}}{c} \\
\ell(j) & =\frac{2}{2-\theta(j)}\left[1+\frac{\theta(j)}{2-\theta(j)} \frac{c_{d}}{c}\right]^{\frac{1}{1-\gamma}} \ell(0) \\
& =y\left(2-\theta_{j}\right)^{\frac{\gamma}{1-\gamma}}\left[\left(2-\theta_{j}\right) c+\theta_{j} c^{d}\right]^{\frac{1}{\gamma-1}} \\
\ell(0) & =\left[\int_{j}\left(\frac{2}{2-\theta}\right)\left(1+\frac{\theta(j)}{2-\theta(j)} \frac{c_{d}}{c}\right)^{\frac{1}{1-\gamma}} d j\right]
\end{aligned}
$$

The last equation exploits the resource constraint on total labor per generation to be one.

Equivalence to the base case without a cost of disability is shown by setting $\chi=0$. We see the disability cost drives a wedge between the relative 
marginal product of labor between two occupations of different risk. Increasing this cost $\chi$ increases the difference in the marginal product of labor between two occupations. The direct implication of this is that increasing these costs decreases relative labor in riskier occupations compared to safer occupations.

In a sense this is a second cost of disability in our model. The first cost was the "human capital" cost of fewer workers given by the fraction $\theta(j)$ who cannot work in the second period. The key difference is that the impact of the human capital cost on the planner's allocation depends on the elasticity of substitution across occupations. For $\gamma>0$, the occupations are gross complements and the planner actually puts more labor in risky occupations relative to a safe ones. The qualitative impact of the utility costs do not depend on this elasticity, but the magnitude of the effect is increasing in the substitutability of occupations.

Step 1: Competitive Equilibrium with Utility Cost of Disability is Pareto Inefficient. In this section we use the result from the previous proposition to argue that the competitive allocation is inefficient both in terms of consumption sharing and occupational sorting.

Proposition B.12 (The Competitive Allocation with Utility Cost of Disability and without Social Insurance is Pareto Inefficient). Let $\left\{c_{j, 1}^{*}, c_{j, n}^{*}, c_{j, d}^{*}\right.$, $\left.a_{j}^{*}, n_{j}^{*}, \ell_{j}^{*}\right\}_{j=1}^{J}$ satisfy the definition of a competitive equilibrium in the case of (i) a continuum of occupations; (ii) cost of disability; (ii) and no social insurance $(b=\tau=0)$. There exists an alternative feasible allocation $\left\{\hat{c}_{j, 1}, \hat{c}_{j, d}, \hat{c}_{j, n}, \hat{\ell}_{j}\right\}_{j=1}^{J}$ that:

(i) increases expected utility in each occupation

$$
\begin{aligned}
& E\left[U_{j}\left(\hat{c}_{j, 1}, \hat{c}_{j, n}, \hat{c}_{j, d}\right)\right] \geq E\left[U_{j}\left(c_{j, 1}^{*}, c_{j, n}^{*}, c_{j, d}^{*}\right)\right] \forall j \in\{1, \ldots, J\} \\
\exists k \text { s.t. } & E\left[U_{k}\left(\hat{c}_{k, 1}, \hat{c}_{k, n}, \hat{c}_{k, d}\right)\right]>E\left[U_{k}\left(c_{k, 1}^{*}, c_{k, n}^{*}, c_{k, d}^{*}\right)\right]
\end{aligned}
$$


(ii) is feasible

$$
\sum_{j} \hat{\ell}_{j}\left(\hat{c}_{j, 1}+\theta_{j} \hat{c}_{j, d}+\left(1-\theta_{j}\right) \hat{c}_{j, n}\right) \leq\left(\sum_{j}\left(\hat{\ell}_{j}\left(2-\theta_{j}\right)\right)^{\gamma}\right)^{\frac{1}{\gamma}}
$$

Proof. This is obvious. We can construct a dominating allocation by fixing labor choices and allowing risk sharing within occupations as shown in the base case. Simply, for any occupation $j>0$, we have $a^{*}(j)>0$ in the competitive equilibrium with incomplete markets. This implies

$$
E_{j} U\left(w^{*}(j)\right)=u\left(w^{*}(j)-a^{*}(j)\right)+\theta(j) u\left(a^{*}(j)\right)+(1-\theta(j)) u\left(w^{*}(j)+a^{*}(j)\right)
$$

Consider instead $\hat{c}_{1}(j)=w^{*}(j)-a^{*}(j)+(1-\theta) a^{*} ; \hat{c}_{d}(j)=a^{*}(j) ;$ and $\hat{c}_{n}(j)=$ $w^{*}(j)$. This is feasible and gives:

$E_{j} U(\hat{c})=u\left(w^{*}(j)-\theta(j) a^{*}(j)\right)+\theta(j) u\left(a^{*}(j)\right)+(1-\theta(j)) u\left(w^{*}(j)\right)>E_{j} U\left(w^{*}(j)\right)$

Step 2: Competitive Equilibrium with Utility Cost of Disability is Productively Inefficient.

Proposition B.13 (The Competitive Allocation with Utility Cost of Disability and without Social Insurance Does not Attain Productive Efficiency of the Complete Markets). Let $\ell^{*}(j)$ satisfy the definition of a competitive equilibrium in the case of (i) a continuum of occupations; (ii) cost of disability; (ii) and no social insurance $(b=\tau=0)$. Let $\ell^{C M}(j)$ be the feasible output maximizing allocation. Then:

$$
y^{*}=\left(\int_{j=0}^{\bar{J}}\left(\ell^{*}(j)\right)^{\gamma} d j\right)^{\frac{1}{\gamma}}<\left(\int_{j=0}^{\bar{J}}\left(\ell^{C M}(j) d j\right)^{\gamma} d j\right)^{\frac{1}{\gamma}}=y^{C M}
$$

ie: the efficient distribution of labor across occupations first-order stochastic dominates the distribution in the competitive equilibrium. 
Proof. This proof follows closely the analogous proof for the baseline model. Suppose, for contradiction that $\exists j>0$ such that:

$$
\frac{\ell(j)}{\ell(0)} \geq \frac{\ell^{C M}(j)}{\ell^{C M}(0)}
$$

Using the definition of competitive wages, this would imply:

$$
\frac{w(j)}{w(0)} \leq \frac{w^{C M}(j)}{w^{C M}(0)}
$$

Since $u()$ is monotone and increasing, this implies:

$$
2 u(w(0))>2 u\left(\frac{w^{C M}(0)}{w^{C M}(j)} w(j)\right.
$$

Let $c_{d}^{C M}=(1+\chi) c^{C M}$. Our assumptions provide $\chi>0$. With algebra on the social planner's optimality of $\ell$ (B.37), we find:

$$
\frac{w_{j}^{C M}}{w_{k}^{C M}}=\frac{2-\theta_{k}}{2-\theta_{j}} \frac{2+\chi \theta_{j}}{2+\chi \theta_{k}}
$$

and in the case of $j=0$ and $k=j$ :

$$
\frac{w_{0}^{C M}}{w_{j}^{C M}}=\frac{2-\theta_{j}}{2+\chi \theta_{j}}
$$

The occupational indifference condition in the competitive equilibrium requires:

$$
\max _{a \geq 0} u(w(j)-a)+\theta(j) u d(a)+(1-\theta(j)) u(w(j)+a)=2 u(w(0))
$$

Yet, we have just shown:

$$
\begin{aligned}
\max _{a \geq 0} u(w(j)-a)+\theta(j) u d(a)+(1-\theta(j)) u(w(j)+a) & =2 u(w(0)) \\
& \geq 2 u\left(\frac{2-\theta_{j}}{2+\chi \theta_{j}} w(j)\right) \\
& >2 u\left(\frac{2-\theta_{j}}{2} w(j)\right)
\end{aligned}
$$


This means the incomplete markets, risky allocation, is strictly preferred to consuming half of expected earnings as a healthy person, in each period. This certainly violates Jensens' inequality and provides a contradiction.

As in the baseline model without cost of disability, we have the corollary that $\frac{\ell(0)}{\ell^{C M}(0)}>1$; the competitive allocation without insurance puts more workers in the risk-free occupation.

Step 3: Comparing the Competitive Allocation of Labor with Utility Cost of Disability to the Complete Markets Allocation..

Proposition B.14 (The Competitive Allocation with Utility Cost of Disability and without Social Insurance Puts too Few Workers in Risky Occupations). Let $\ell^{*}(j)$ satisfy the definition of a competitive equilibrium in the case of (i) a continuum of occupations; (ii) cost of disability; (ii) and no social insurance $(b=\tau=0)$. Let $\ell^{c m}(j)$ be the feasible output maximizing allocation. Then:

$$
\int_{j=0}^{t} \ell^{*}(j) d j \leq \int_{j=0}^{t} \ell^{c m}(j) d j \quad \forall t \in[0, J]
$$

ie: the efficient distribution of labor across occupations first-order stochastic dominates the distribution in the competitive equilibrium.

Proof. This proof follows closely the analogous proof for the baseline model, Proof B.4. Suppose, for contradiction that $\exists j<k$ such that:

$$
\frac{\ell^{*}(k)}{\ell^{*}(j)} \geq \frac{\ell^{C M}(k)}{\ell^{C M}(j)}
$$

Using the definition of competitive wages, this would imply:

$$
\frac{w(k)}{w(j)} \leq \frac{w^{C M}(k)}{w^{C M}(j)}
$$


and since $\frac{w_{j}^{C M}}{w_{k}^{C M}}=\frac{2-\theta_{k}}{2-\theta_{j}} \frac{2+\chi \theta_{j}}{2+\chi \theta_{k}}$ :

$$
w^{*}(k)(2-\theta(k))(2+\theta(j) \chi)<w^{*}(j)(2-\theta(j))(2+\theta(k) \chi)
$$

Since $\chi>0$ and we are given $\theta(j)<\theta(k)$, the result is that the total expected income in occupation $k$ is less than in occupation $j$

$$
w^{*}(k)(2-\theta(k))<w^{*}(j)(2-\theta(j))
$$

We have reduced the problem to the baseline case. Together we have lower expected income and higher risk in occupation $k$. This arrangement cannot satisfy indifference in expected utilities across occupations. See proof for the baseline case for a formal exposition.

Step 4: Welfare Gain from SDI w/ Costly Disability. The prior two steps have shown the competitive allocation of the economy with costly disability generates the same sources of inefficiency, lack of risk sharing and productive inefficiency, as the baseline economy. In this final step we show the marginal introduction of social disability insurance (SDI) continues to increase welfare.

Proposition B.15 (Social Insurance is Welfare Improving (on the margin)). Let $E U(\tau)$ be the expected utility of an agent from the competitive equilibrium in the case of (i) a continuum of occupations; (ii) costly disability; and (iii) and proportional social insurance $\left(\tau \geq 0, b_{j}=b w_{j}\right.$, and $\sum_{j}\left(2-\theta_{j}\right) \ell_{j} \tau w_{j}=$ $\left.\sum_{j} \theta_{j} \ell_{j} b w_{j}\right)$. Then:

$$
\frac{d E U(\tau)}{d \tau} \|_{\tau=0}>0
$$

Proof. Lemma 1. SDI increases output $\left(\left.\frac{d y^{*}}{d \tau}\right|_{\tau=0}>0\right)$. First, we will show:

$$
\frac{d}{d \tau}\left(\frac{\ell^{*}(j, \tau)}{\ell^{*}(0, \tau)}\right)>0 \quad \forall j>0
$$

Following the analogous proof for the baseline case, we consider $\delta$ such that $\forall \epsilon \in(0, \delta)$, we have $a^{*}(j, \delta)>0$ for every $j>0$. That is to say, the 
borrowing constraint does not bind and choices are all in the interior of the budget set.

Hold wages constant at the no-insurance competitive level. Then for $j>0$ the expected income at $\tau=0$ and $\tau=\epsilon>0$ are $(2-\theta(j)) w(j, 0)$ and $(2-\theta(j)) w(j, 0)(1-\epsilon)+\theta(j) \hat{b}(\epsilon \mid 0) w(j, 0)$, respectively. We define $b(\epsilon)$ as the level of benefits implied by $\tau=\epsilon$ and $w(j, \epsilon)$.

The expected income at $\tau=\epsilon>0$ translates to higher expected consumption under our assumption $u^{\prime}(c)=u^{\prime d}((1+\chi) c)$ for all $c$, if $\frac{b(\epsilon)}{1+\chi}>\epsilon$. Since we restrict $\theta(j) \leq \frac{1}{2}$ we have at most $\frac{1}{4}$ of the population in each occupation disabled. Considering repayments only within occupation we would have a dependency ratio of 1-to-3 implying $b \geq 3 \tau$. Therefore, a sufficient (although not necessary) condition for the remainder of our proofs to go through is:

$$
(1+\chi)<3 \Rightarrow \frac{3 \tau}{1+\chi}>\tau \rightarrow \frac{b(\tau)}{1+\chi}>\tau
$$

With this result, we apply the Maximum Theorem as in the baseline model to show expected utility is higher:

$$
\begin{gathered}
\max _{a_{\epsilon}} u\left(w(j, 0)(1-\epsilon)-a_{\epsilon}\right)+\theta(j) u^{d}\left(w(j, 0) b(\epsilon)+a_{\epsilon}\right)+(1-\theta(j)) u\left(w(j, 0)(1-\epsilon)+a_{\epsilon}\right) \\
>\max _{a_{0}} u\left(w(j, 0)-a_{0}\right)+\theta(j) u^{d}\left(a_{0}\right)+(1-\theta(j)) u\left(w(j, 0)+a_{0}\right)
\end{gathered}
$$

For $j=0$, however, the expected utility is lower with the $\epsilon$ tax rate:

$$
2 u(w(0,0)(1-\epsilon))<2 u(w(0,0))
$$

Based on indifference under $\tau=0$, we have

$$
\begin{aligned}
\max _{a_{\epsilon}} u\left(w(j, 0)(1-\epsilon)-a_{\epsilon}\right)+\theta(j) u^{d}\left(w(j, 0) b(\epsilon)+a_{\epsilon}\right)+(1-\theta(j)) u\left(w(j, 0)(1-\epsilon)+a_{\epsilon}\right) \\
>2 u(w(0,0)(1-\epsilon))
\end{aligned}
$$

This violates the indifference condition of the competitive equilibrium with $\tau=\epsilon$ and so we must have a change in both the wage in the $j=0$ 
occupation and the relative wage in the $j>0$ occupations: $w(j, \epsilon) \neq w(j, 0)$ and $\frac{w(j, \epsilon)}{w(0, \epsilon)} \neq \frac{w(j, 0)}{w(0,0)}$

The remainder of the proof showing it must be $w(j, \epsilon)<w(j, 0)$ and $\frac{w(j, \epsilon)}{w(0, \epsilon)}<\frac{w(j, 0)}{w(0,0)}$ follows directly the proof for the baseline case and so is omitted.

Corollary 1. Marginal SDI decreases labor in the risk free occupation: $\ell^{*}(0, \epsilon)<\ell^{*}(0,0)$ This follows a direct application of Corollary E.6, the analogous corollary for the baseline case.

Lemma 2. Output is increasing at the marginal introduction of SDI: $\left.\frac{d y^{*}(\tau)}{d \tau}\right|_{\tau=0}>0$ This follows a direct application of Lemma E.7, the analogous lemma for the baseline case.

Part 2. SDI increases welfare: $\left.\frac{d E U(j, \tau)}{d \tau}\right|_{\tau=0}>0$ for all $j$

This proof again follows from the baseline case once adjusting disability benefits to the non-disabled equivalent consumption: $\hat{b}(\tau)=\frac{b(\tau)}{1+\chi}$ and proceeding from there.

\section{B.10. Extension: Non-verifiable disability status}

Proposition B.16 (The Optimal SDI Policy is Robust to Non-Verifiable Disability Status $)$. Let $\left\{c_{1}^{*}\left(j, \tau^{*}\right), c_{n}^{*}\left(j, \tau^{*}\right), c_{d}^{*}\left(j, \tau^{*}\right), a^{*}\left(j, \tau^{*}\right), w\left(j, \tau^{*}\right)\right\}_{j=1}^{J}$ satisfy the definition of a competitive equilibrium at the optimal SDI policy $(\tau, b=b(\tau))$. Then, the expected utility of reporting disability status truthfully, $\operatorname{EU}(j, \tau)$, is greater than any time-consistent deviation:

$$
\begin{gathered}
E U(j, \tau)=\max _{a} u((1-\tau) w(j, \tau)-a)+\theta(j) u(b w(j, \tau)+a)+(1-\theta(j)) u((1-\tau) w(j, \tau)+a) \\
\geq \max _{a} u((1-\tau) w(j, \tau)-a)+\theta(j) u(b w(j, \tau)+a) \\
\quad+(1-\theta(j)) \max \{u((1-\tau) w(j, \tau)+a), u(b w(j, \tau)+a)\}
\end{gathered}
$$

Proof. We first show that a worker in any occupation $j \in[0, J]$ who enters the second period with an arbitrary asset $a>0$ optimizes by truthfully 
revealing their disability status when healthy, ie:

$$
u(b w(j, \tau)+a) \leq u(w(j, \tau)(1-\tau)+a)
$$

where the left-hand side is the payoff if the worker claims disability and right-hand side is the payoff from working. This holds if $b \leq 1-\tau$, which we have shown in Lemma B.4 to be the case given our restriction $J \leq \frac{1}{2}$. We can quickly recap that result here: From the government budget constraint, given a $\tau, b$ satisfies

$$
b \int_{k} \theta(k) w(k, \tau) d k=\tau \int_{k}(2-\theta(k)) w(k, \tau) d k
$$

and therefore $b=\tau \frac{\int_{k}(2-\theta(k)) w(k, \tau) d k}{\int_{k} \theta(k) w(k, \tau) d k} . \theta(k)<1$ and $w(k, \tau)>0 \forall k, \frac{\int_{k}(2-\theta(k)) w(k, \tau) d k}{\int_{k} \theta(k) w(k, \tau) d k}<$ 1 and thus $b \geq \tau$ and equivalently $1-b \leq 1-\tau$.

Finally, if $b<\frac{1}{2}$ then $b \leq 1-b$ and $1-b \leq 1-\tau \Rightarrow b \leq 1-\tau$.

Therefore, any time consistent plan requires truth-telling in the final period and so the agent cannot commit to lie from period 1 . 


\section{List of references}

Acemoglu, D., Shimer, R., October 1999. Efficient unemployment insurance. Journal of Political Economy 107 (5), 893-928.

Administration, S. S., 2017. Annual statistical report on the social security disability insurance program, 2016. Tech. Rep. 1311826, Office of Retirement and Disability Policy: Office of Research, Evaluation and Statistics.

Duggan, M., Autor, D., 2006. The growth in the social security disability rolls: a fiscal crisis unfolding. Tech. Rep. 12436, National Bureau of Economic Research.

Fletcher, J. M., Sindelar, J. L., Yamaguchi, S., June 2009. Cumulative effects of job characteristics on health. Working Paper 15121, National Bureau of Economic Research.

Golosov, M., Tsyvinski, A., 2006. Designing optimal disability insurance: A case for asset testing. Journal of Political Economy 114 (2), 257-279.

Groshen, E. L., Perez, T. E., September 2015. National compensation survey: Employee benefits in the united states, march 2015. Bulletin 2782, U.S. Bureau of Labor Statistics.

Health and Retirement Study, 2013. HRS Core public use dataset. Produced and distributed by the University of Michigan with funding from the National Institute on Aging (grant number NIA U01AG009740). Ann Arbor, MI.

Isenberg, K. N., 2016. U.s. individual disability income insurance 2015 annual supplement. Tech. rep., LIMRA.

Katz, L. F., Autor, D., 1999. Changes in the wage structure and earnings inequality. Handbook of labor economics 3, 1463-1555. 
Low, H., Meghir, C., Pistaferri, L., 2015. Disability insurance and the dynamics of the incentive-insurance tradeoff. American Economic Review.

Michaud, A. M., Wiczer, D., Sep. 2017. The Disability Option: Labor Market Dynamics with Macroeconomic and Health Risks. Tech. rep., Mimeo.

Morefield, G. B., Ribar, D. C., Ruhm, C. J., February 2011. Occupational status and health transitions. Working Paper 16794, National Bureau of Economic Research.

RAND HRS Data, Version P, August 2016. Produced by the RAND Center for the Study of Aging, with funding from the National Institute on Aging and the Social Security Administration. Santa Monica, CA.

Ravesteijn, B., van Kippersluis, H., van Doorslaer, E., Sep. 2013. The Wear and Tear on Health: What is the Role of Occupation? MPRA Paper 50321, University Library of Munich, Germany.

Schulhofer-Wohl, S., 2011. Heterogeneity and Tests of Risk Sharing. Journal of Political Economy 119 (5), 925 - 958. 


\section{List of Figures}

1 The density across occupations of the incidence of difficulties with ADLs $\ldots \ldots \ldots \ldots \ldots \ldots \ldots$

2 The competitive allocation relative to the efficient, planner's at vaious levels of risk aversion. The labor allocation diverges more greatly from the optimal planner's at higher levels of risk aversion. . . . . . . . . . . . . . . . .

3 The competitive allocations relative to the efficient at various elasticities of substitution. Distortions away from the optimal planner's increase in $\gamma$ to a point, then at infinity they again converge. The optimal allocation places more in the risky occupatiion when they are gross complements and more in the safe when they are substitues. . . . . . . . . . . .

4 Output, disability, and welfare for different tax levels. The peak output is achieved at a lower tax than the welfare maximizing tax. . . . . . . . . . . .

5 The welfare effect of SDI on different groups. Among lowrisk occupations, the benefit comes from increased output and therefore wages, which is seen in ex post gains. High-risk occupations gain in an ex ante sense from risk sharing. . . . .

6 As the price of disability insurance increases, the contracts remain unprofitable (Left) and the risk pool deteriorates (Right). On left, Revenue and outlays ofcontracts are normalized to their fraction of SDI with the same price $(\operatorname{tax}) . \ldots \ldots$

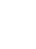
5

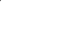




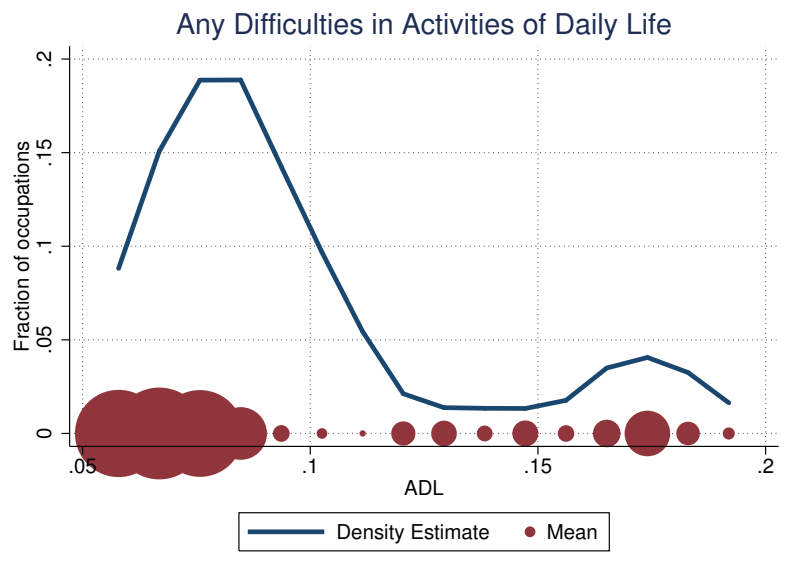

Figure 1: The density across occupations of the incidence of difficulties with ADLs 

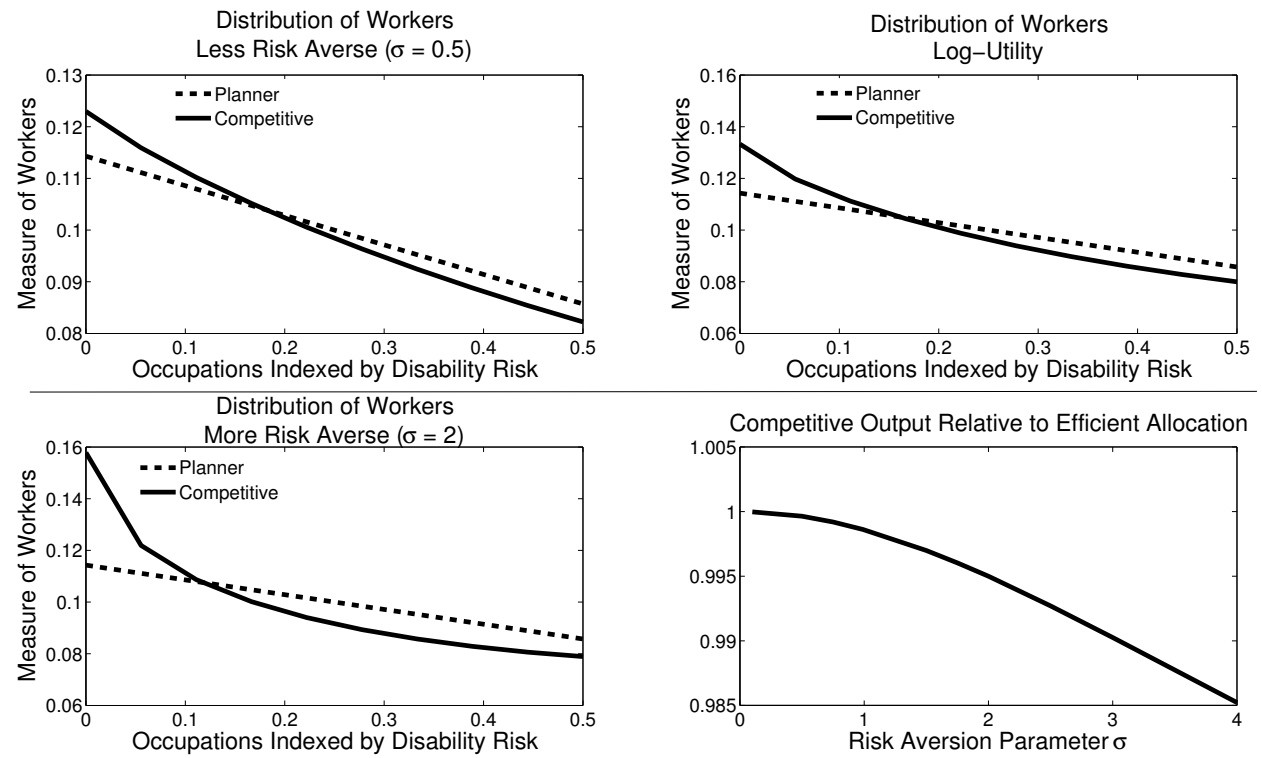

Figure 2: The competitive allocation relative to the efficient, planner's at vaious levels of risk aversion. The labor allocation diverges more greatly from the optimal planner's at higher levels of risk aversion. 

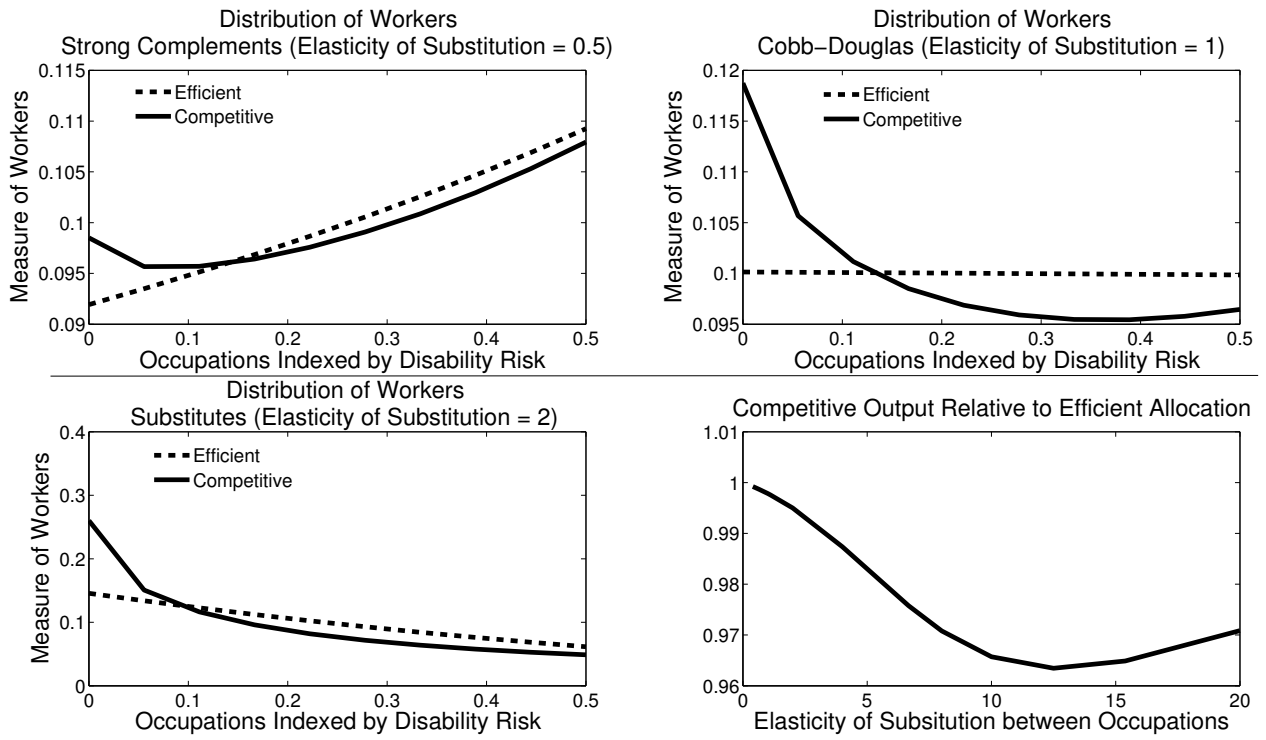

Figure 3: The competitive allocations relative to the efficient at various elasticities of substitution. Distortions away from the optimal planner's increase in $\gamma$ to a point, then at infinity they again converge. The optimal allocation places more in the risky occupatiion when they are gross complements and more in the safe when they are substitues. 

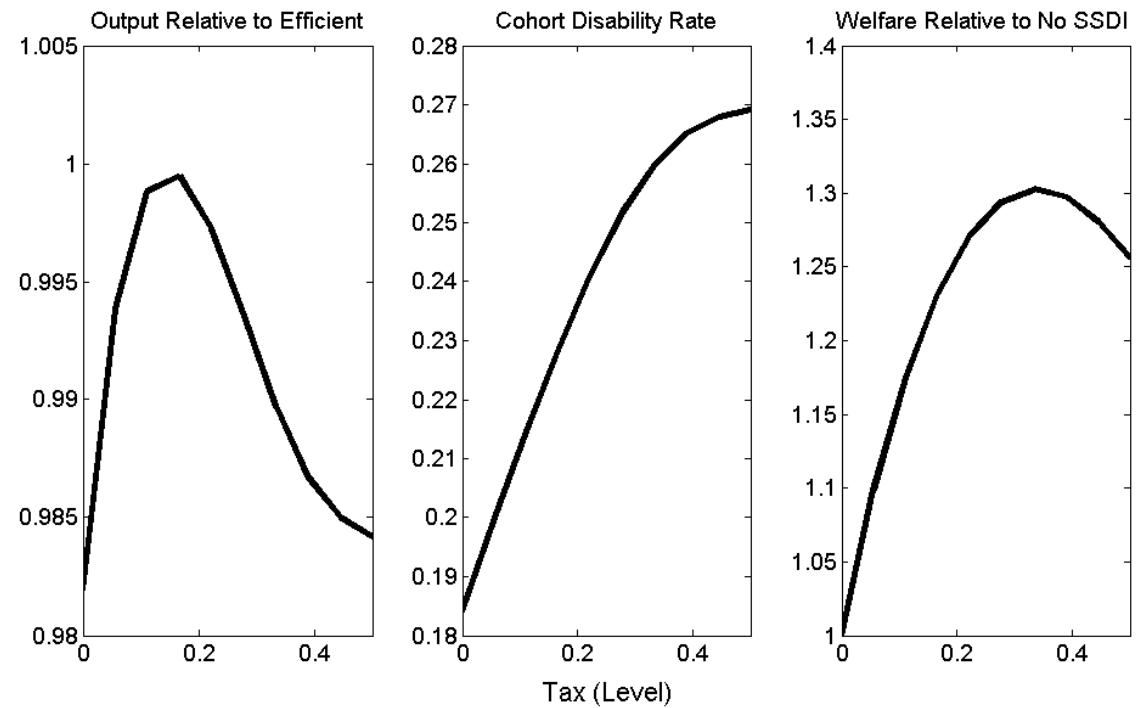

Figure 4: Output, disability, and welfare for different tax levels. The peak output is achieved at a lower tax than the welfare maximizing tax. 

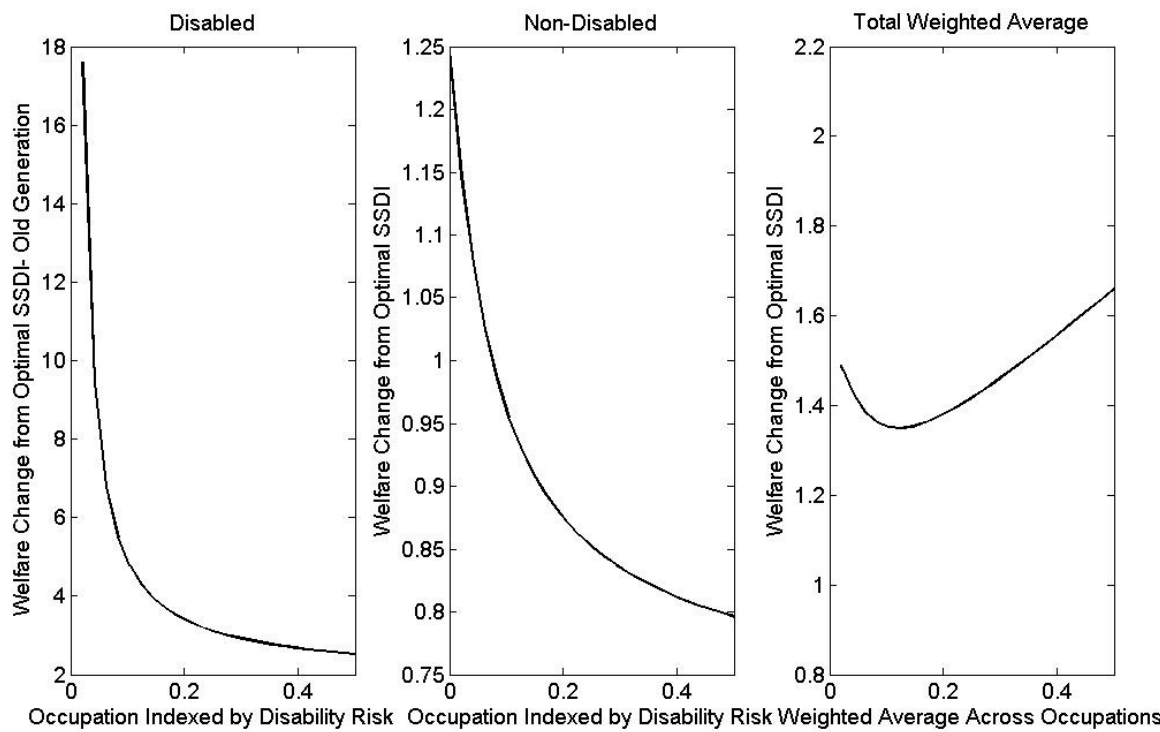

Figure 5: The welfare effect of SDI on different groups. Among low-risk occupations, the benefit comes from increased output and therefore wages, which is seen in ex post gains. High-risk occupations gain in an ex ante sense from risk sharing. 

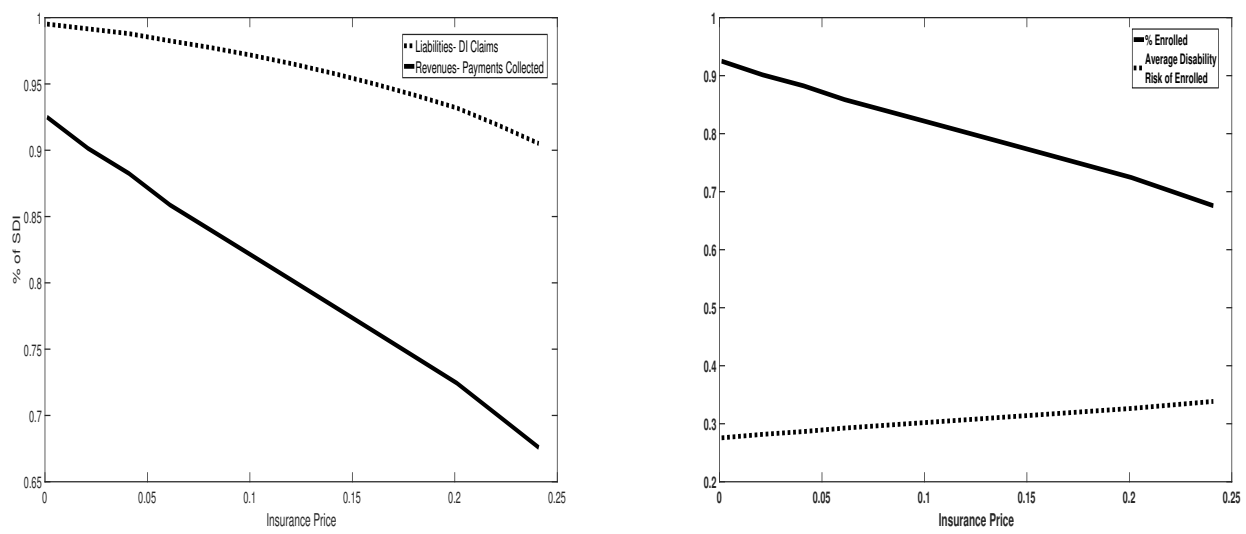

Figure 6: As the price of disability insurance increases, the contracts remain unprofitable (Left) and the risk pool deteriorates (Right). On left, Revenue and outlays ofcontracts are normalized to their fraction of SDI with the same price (tax). 


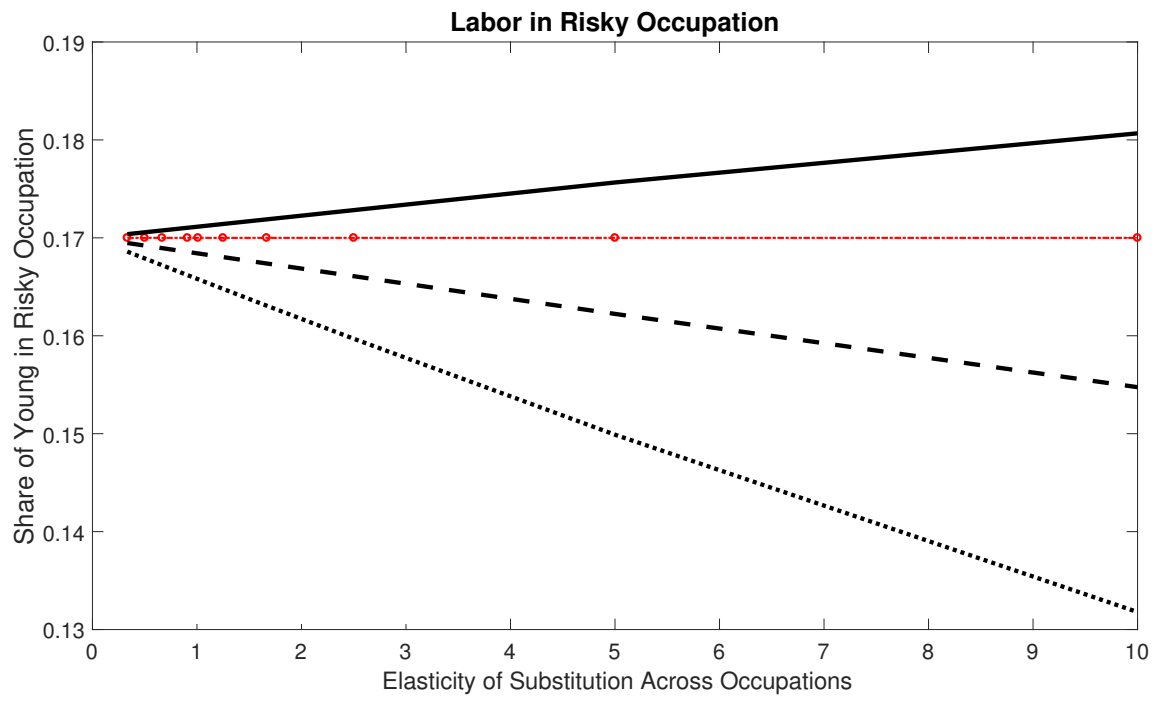

Figure 7: Labor in the risky occupation is higher under the baseline and constrained optimal SDI programs than the output maximizing allocation. It is lower with only actuarially fair DI. 


\section{List of Tables}

1 The decomposition of the occupation-group effect on disability. Occupations are split between low- and high-risk and the regressors are a cubic for potential experience, body mass index (BMI), time, dummies for education level, gender, marital status, race, and tobacco use. Column (1) uses only one observation per individual and (2) pools all of the data. . . . . . 12

2 The effect of an occupation's physical requirements on workinglife disability. Columns (1) - (3) use our instrumental variable schemes and Columns (4) - (6) treat physical requirements as exogenous. (1) and (3) are marginal effects at the mean from probit models. (3) and (6) use a self-reported health limitation as the dependent variable rather than an ADL difficulty

3 The optimal system compared to observed US system, constrained optimal policy, and actuarially fair in a calibrated model ...................... . . 14

4 First stage estimates using non-physical descriptors to instrument physical requirements. . . . . . . . . . . . . 15

$5 \quad$ First stage estimates using non-physical descriptors to instrument physical requirements. We include industry fixed effects and clustering standard errors on industry . . . . . . . . . . . 16

6 LPM estimate of the effect of an occupation's physical requirements on working-life disability. Columns (1) - (3) use our instrumental variable schemes and Columns (4) - (6) treat physical requirements as exogenous. (1) and (3) are marginal effects at the mean from probit models. (3) and (6) use a selfreported health limitation as the dependent variable rather than an ADL difficulty . . . . . . . . . . . . . 17 


\begin{tabular}{lcc}
\hline & $(1)$ & $(2)$ \\
\hline Safe & 0.189 & 0.106 \\
Risky & 0.264 & 0.157 \\
Difference & -0.076 & -0.051 \\
\hline Observables & -0.044 & -0.030 \\
$\quad$ \% Difference & 57.9 & 58.8 \\
\hline Occupation & -0.031 & -0.021 \\
$\quad$ \% Difference & 42.1 & 41.2 \\
\hline$N$ & 20,328 & 127,298 \\
\hline
\end{tabular}

Table 1: The decomposition of the occupation-group effect on disability. Occupations are split between low- and high-risk and the regressors are a cubic for potential experience, body mass index (BMI), time, dummies for education level, gender, marital status, race, and tobacco use. Column (1) uses only one observation per individual and (2) pools all of the data. 


\begin{tabular}{|c|c|c|c|c|c|c|}
\hline & $(1)$ & $(2)$ & $(3)$ & (4) & $(5)$ & (6) \\
\hline O*NET Phys & $\begin{array}{c}0.034^{* *} \\
(0.005)\end{array}$ & $\begin{array}{c}0.035^{* *} \\
(0.010)\end{array}$ & $\begin{array}{c}0.013^{* *} \\
(0.004)\end{array}$ & $\begin{array}{c}0.031^{* *} \\
(0.005)\end{array}$ & $\begin{array}{c}0.033^{* *} \\
(0.010)\end{array}$ & $\begin{array}{c}0.012^{* *} \\
(0.004)\end{array}$ \\
\hline Experience & $\begin{array}{c}0.033^{* *} \\
(0.001)\end{array}$ & $\begin{array}{c}0.033^{* *} \\
(0.001)\end{array}$ & $\begin{array}{c}0.005^{* *} \\
(0.001)\end{array}$ & $\begin{array}{c}0.033^{* *} \\
(0.001)\end{array}$ & $\begin{array}{c}0.033^{* *} \\
(0.001)\end{array}$ & $\begin{array}{c}0.005^{* *} \\
(0.001)\end{array}$ \\
\hline BMI & $\begin{array}{c}0.012^{* *} \\
(0.001)\end{array}$ & $\begin{array}{c}0.012^{* *} \\
(0.001)\end{array}$ & $\begin{array}{c}0.003^{* *} \\
(0.001)\end{array}$ & $\begin{array}{c}0.012^{* *} \\
(0.001)\end{array}$ & $\begin{array}{c}0.012^{* *} \\
(0.001)\end{array}$ & $\begin{array}{c}0.003^{* *} \\
(0.001)\end{array}$ \\
\hline Woman & $\begin{array}{c}0.027^{* *} \\
(0.009)\end{array}$ & $\begin{array}{c}0.032^{* *} \\
(0.011)\end{array}$ & $\begin{array}{l}-0.003 \\
(0.007)\end{array}$ & $\begin{array}{c}0.026^{* *} \\
(0.008)\end{array}$ & $\begin{array}{c}0.031^{* *} \\
(0.011)\end{array}$ & $\begin{array}{c}-0.003 \\
(0.007)\end{array}$ \\
\hline Self-employed & $\begin{array}{c}-0.022 * \\
(0.010)\end{array}$ & $\begin{array}{c}-0.022^{\dagger} \\
(0.013)\end{array}$ & $\begin{array}{c}-0.029^{* *} \\
(0.008)\end{array}$ & $\begin{array}{c}-0.022^{*} \\
(0.010)\end{array}$ & $\begin{array}{c}-0.022^{\dagger} \\
(0.013)\end{array}$ & $\begin{array}{c}-0.029^{* *} \\
(0.008)\end{array}$ \\
\hline College & $\begin{array}{c}0.144^{* *} \\
(0.010)\end{array}$ & $\begin{array}{c}0.148^{* *} \\
(0.013)\end{array}$ & $\begin{array}{c}-0.033^{* *} \\
(0.008)\end{array}$ & $\begin{array}{c}0.142 * * \\
(0.010)\end{array}$ & $\begin{array}{c}0.147^{* *} \\
(0.013)\end{array}$ & $\begin{array}{c}-0.034^{* *} \\
(0.008)\end{array}$ \\
\hline No HS & $\begin{array}{c}-0.093^{* *} \\
(0.011)\end{array}$ & $\begin{array}{c}-0.092^{* *} \\
(0.011)\end{array}$ & $\begin{array}{c}0.045^{* *} \\
(0.008)\end{array}$ & $\begin{array}{c}-0.092^{* *} \\
(0.011)\end{array}$ & $\begin{array}{c}-0.091^{* *} \\
(0.011)\end{array}$ & $\begin{array}{c}0.045^{* *} \\
(0.008)\end{array}$ \\
\hline Not married & $\begin{array}{c}0.087^{* *} \\
(0.011)\end{array}$ & $\begin{array}{c}0.085^{* *} \\
(0.009)\end{array}$ & $\begin{array}{l}0.015^{\dagger} \\
(0.009)\end{array}$ & $\begin{array}{c}0.087^{* *} \\
(0.011)\end{array}$ & $\begin{array}{c}0.085^{* *} \\
(0.009)\end{array}$ & $\begin{array}{l}0.016^{\dagger} \\
(0.009)\end{array}$ \\
\hline Not white & $\begin{array}{c}0.031^{* *} \\
(0.010)\end{array}$ & $\begin{array}{c}0.033^{* *} \\
(0.012)\end{array}$ & $\begin{array}{c}-0.019 * \\
(0.008)\end{array}$ & $\begin{array}{c}0.031^{* *} \\
(0.010)\end{array}$ & $\begin{array}{c}0.034^{* *} \\
(0.012)\end{array}$ & $\begin{array}{c}-0.019 * \\
(0.008)\end{array}$ \\
\hline Smoker & $\begin{array}{l}0.021 * \\
(0.009)\end{array}$ & $\begin{array}{l}0.020^{\dagger} \\
(0.011)\end{array}$ & $\begin{array}{c}0.020^{* *} \\
(0.007)\end{array}$ & $\begin{array}{l}0.021 * \\
(0.009)\end{array}$ & $\begin{array}{l}0.021^{\dagger} \\
(0.011)\end{array}$ & $\begin{array}{c}0.020^{* *} \\
(0.007)\end{array}$ \\
\hline Industry FE & & $\mathrm{X}$ & & & $\mathrm{X}$ & \\
\hline Observations & 14763 & 14558 & 14763 & 14763 & 14558 & 14763 \\
\hline
\end{tabular}

Table 2: The effect of an occupation's physical requirements on working-life disability. Columns (1) - (3) use our instrumental variable schemes and Columns (4) - (6) treat physical requirements as exogenous. (1) and (3) are marginal effects at the mean from probit models. (3) and (6) use a self-reported health limitation as the dependent variable rather than an ADL difficulty . 


\begin{tabular}{|c|c|c|c|}
\hline & \multicolumn{3}{|c|}{ Elasticity of Substitution } \\
\hline & & 2 & \\
\hline Young Labor in Risky Occupation & & & \\
\hline Baseline & $17 \%$ & $17 \%$ & $17 \%$ \\
\hline Constrained Optimal & $17.17 \%$ & $18.75 \%$ & $21.59 \%$ \\
\hline Actuarially Fair & $16.90 \%$ & $16.02 \%$ & $14.63 \%$ \\
\hline $\begin{array}{l}\text { Welfare Gain from Constrained Optimal DI } \\
\text { (consumption equiv rel. to Baseline) }\end{array}$ & $+104 \%$ & $+104 \%$ & +10307 \\
\hline (consumption equiv rel. to Actuarially Fair ) & $\begin{array}{l}+1.04 \% \\
+6.3 \%\end{array}$ & $+6.3 \%$ & $\begin{array}{l}+1.0 .0 \% \\
+6.2 \%\end{array}$ \\
\hline Constrained Optimal Output & & & \\
\hline (rel. to Baseline) & $+0.00 \%$ & $+0.00 \%$ & $+0.00 \%$ \\
\hline (rel. to Actuarially Fair ) & $+0.00 \%$ & $+0.06 \%$ & $+0.12 \%$ \\
\hline $\begin{array}{l}\text { Aggregate Disability } \\
\text { (rel. to baseline) }\end{array}$ & $+0.14 \%$ & $+1.50 \%$ & $+3.92 \%$ \\
\hline (rel. to Actuarially Fair ) & $+0.23 \%$ & $+2.34 \%$ & $+5.95 \%$ \\
\hline Replacement rate & & & \\
\hline Baseline & $40.00 \%$ & $40.00 \%$ & $40.00 \%$ \\
\hline Constrained Optimal & $121.13 \%$ & $120.68 \%$ & $118.95 \%$ \\
\hline Tax rate & & & \\
\hline Baseline & $1.59 \%$ & $1.59 \%$ & $1.59 \%$ \\
\hline Constrained Optimal & $4.80 \%$ & $4.85 \%$ & $4.90 \%$ \\
\hline
\end{tabular}

Table 3: The optimal system compared to observed US system, constrained optimal policy, and actuarially fair in a calibrated model 


\begin{tabular}{lcc}
\hline \hline O*NET Non-phys Reqs, PC1 & $-0.712^{* *}$ & $(0.00211)$ \\
O*NET Non-phys Reqs, PC2 & $0.269^{* *}$ & $(0.00335)$ \\
& & \\
O*NET Non-phys Reqs, PC3 & $0.302^{* *}$ & $(0.00261)$ \\
& & \\
Constant & $-0.00901^{* *}$ & $(0.00249)$ \\
\hline Observations & 14763 & \\
$R^{2}$ & 0.933 & \\
$\mathrm{~F}$ & 107126.6 & \\
\hline Robust standard errors in parentheses \\
$\dagger p<0.10,{ }^{*} p<0.05,{ }^{* *} p<0.01$
\end{tabular}

Table 4: First stage estimates using non-physical descriptors to instrument physical requirements. 


\begin{tabular}{lcc}
\hline \hline O*NET Non-phys Reqs, PC1 & $-0.711^{* *}$ & $(0.00212)$ \\
O*NET Non-phys Reqs, PC2 & $0.269^{* *}$ & $(0.00336)$ \\
O*NET Non-phys Reqs, PC3 & $0.301^{* *}$ & $(0.00261)$ \\
& & \\
Constant & $-0.00922^{* *}$ & $(0.00251)$ \\
\hline Observations & 14558 & \\
$R^{2}$ & 0.933 & \\
F & 104812.0 & \\
\hline Robust standard errors in parentheses & \\
${ }^{\dagger} p<0.10,{ }^{*} p<0.05,{ }^{* *} p<0.01$ & &
\end{tabular}

Table 5: First stage estimates using non-physical descriptors to instrument physical requirements. We include industry fixed effects and clustering standard errors on industry 


\begin{tabular}{|c|c|c|c|c|c|c|}
\hline & $(1)$ & $(2)$ & $(3)$ & (4) & (5) & (6) \\
\hline O*NET Phys & $\begin{array}{c}0.037^{* *} \\
(0.005)\end{array}$ & $\begin{array}{c}0.038^{* *} \\
(0.010)\end{array}$ & $\begin{array}{c}0.013^{* *} \\
(0.004)\end{array}$ & $\begin{array}{c}0.034^{* *} \\
(0.005)\end{array}$ & $\begin{array}{c}0.036^{* *} \\
(0.011)\end{array}$ & $\begin{array}{c}0.012 \text { ** } \\
(0.004)\end{array}$ \\
\hline Experience & $\begin{array}{c}0.032 \text { ** } \\
(0.001)\end{array}$ & $\begin{array}{c}0.0322^{* *} \\
(0.001)\end{array}$ & $\begin{array}{c}0.005^{* *} \\
(0.001)\end{array}$ & $\begin{array}{c}0.032 \text { ** } \\
(0.001)\end{array}$ & $\begin{array}{c}0.032 \text { ** } \\
(0.001)\end{array}$ & $\begin{array}{c}0.005^{* *} \\
(0.001)\end{array}$ \\
\hline BMI & $\begin{array}{c}0.015^{* *} \\
(0.001)\end{array}$ & $\begin{array}{c}0.015^{* *} \\
(0.001)\end{array}$ & $\begin{array}{c}0.003^{* *} \\
(0.001)\end{array}$ & $\begin{array}{c}0.015^{* *} \\
(0.001)\end{array}$ & $\begin{array}{c}0.015^{* *} \\
(0.001)\end{array}$ & $\begin{array}{c}0.003^{* *} \\
(0.001)\end{array}$ \\
\hline Woman & $\begin{array}{c}0.046^{* *} \\
(0.008)\end{array}$ & $\begin{array}{c}0.0511^{* *} \\
(0.011)\end{array}$ & $\begin{array}{c}-0.002 \\
(0.007)\end{array}$ & $\begin{array}{c}0.045^{* *} \\
(0.008)\end{array}$ & $\begin{array}{c}0.050^{* *} \\
(0.012)\end{array}$ & $\begin{array}{l}-0.002 \\
(0.007)\end{array}$ \\
\hline Self-employed & $\begin{array}{l}-0.014 \\
(0.009)\end{array}$ & $\begin{array}{c}-0.014 \\
(0.013)\end{array}$ & $\begin{array}{c}-0.027^{* *} \\
(0.007)\end{array}$ & $\begin{array}{l}-0.014 \\
(0.009)\end{array}$ & $\begin{array}{c}-0.014 \\
(0.014)\end{array}$ & $\begin{array}{c}-0.027^{* *} \\
(0.007)\end{array}$ \\
\hline College & $\begin{array}{c}0.154^{* *} \\
(0.010)\end{array}$ & $\begin{array}{c}0.160^{* *} \\
(0.014)\end{array}$ & $\begin{array}{c}-0.027^{* *} \\
(0.008)\end{array}$ & $\begin{array}{c}0.153^{* *} \\
(0.010)\end{array}$ & $\begin{array}{c}0.159^{* *} \\
(0.014)\end{array}$ & $\begin{array}{c}-0.027^{* *} \\
(0.008)\end{array}$ \\
\hline No HS & $\begin{array}{c}-0.083^{* *} \\
(0.011)\end{array}$ & $\begin{array}{c}-0.084^{* *} \\
(0.012)\end{array}$ & $\begin{array}{c}0.059^{* *} \\
(0.010)\end{array}$ & $\begin{array}{c}-0.082^{* *} \\
(0.011)\end{array}$ & $\begin{array}{c}-0.084^{* *} \\
(0.012)\end{array}$ & $\begin{array}{c}0.059^{* *} \\
(0.010)\end{array}$ \\
\hline Not married & $\begin{array}{c}0.096^{* *} \\
(0.013)\end{array}$ & $\begin{array}{c}0.094^{* *} \\
(0.011)\end{array}$ & $\begin{array}{l}0.016^{\dagger} \\
(0.009)\end{array}$ & $\begin{array}{c}0.096^{* *} \\
(0.013)\end{array}$ & $\begin{array}{c}0.094^{* *} \\
(0.011)\end{array}$ & $\begin{array}{l}0.016^{\dagger} \\
(0.009)\end{array}$ \\
\hline Not white & $\begin{array}{c}0.041^{* *} \\
(0.012)\end{array}$ & $\begin{array}{c}0.044^{* *} \\
(0.014)\end{array}$ & $\begin{array}{c}-0.018 * \\
(0.009)\end{array}$ & $\begin{array}{c}0.042^{* *} \\
(0.012)\end{array}$ & $\begin{array}{l}0.045 * \\
(0.015)\end{array}$ & $\begin{array}{c}-0.018^{*} \\
(0.009)\end{array}$ \\
\hline Smoker & $\begin{array}{c}0.025^{* *} \\
(0.009)\end{array}$ & $\begin{array}{l}0.024^{\dagger} \\
(0.013)\end{array}$ & $\begin{array}{l}0.020 * \\
(0.008)\end{array}$ & $\begin{array}{c}0.025^{* *} \\
(0.009)\end{array}$ & $\begin{array}{l}0.025^{\dagger} \\
(0.013)\end{array}$ & $\begin{array}{l}0.020 * \\
(0.008)\end{array}$ \\
\hline Industry FE & & $\mathrm{X}$ & & $\mathrm{X}$ & & \\
\hline Observations & 14763 & 14558 & 14763 & 14763 & 14558 & 14763 \\
\hline
\end{tabular}

Table 6: LPM estimate of the effect of an occupation's physical requirements on workinglife disability. Columns (1) - (3) use our instrumental variable schemes and Columns (4) (6) treat physical requirements as exogenous. (1) and (3) are marginal effects at the mean from probit models. (3) and (6) use a self-reported health limitation as the dependent variable rather than an ADL difficulty . 